\title{
Pair-Density-Wave Order and Paired Fractional Quantum Hall Fluids
}

\author{
Luiz H. Santos, ${ }^{1,2, *}$ Yuxuan Wang, ${ }^{1,3,{ }^{*}}$ and Eduardo Fradkin ${ }^{1}$ \\ ${ }^{1}$ Department of Physics and Institute for Condensed Matter Theory, \\ University of Illinois at Urbana-Champaign, 1110 West Green Street, \\ Urbana, Illinois, 61801-3080, USA \\ ${ }^{2}$ Department of Physics, Emory University, 400 Dowman Drive, Atlanta, Georgia 30322, USA \\ ${ }^{3}$ Department of Physics, University of Florida, 2001 Museum Road, Gainesville, Florida 32611, USA
}

(Received 30 November 2018; published 5 June 2019)

\begin{abstract}
The properties of the isotropic incompressible $\nu=5 / 2$ fractional quantum Hall $(\mathrm{FQH})$ state are described by a paired state of composite fermions in zero (effective) magnetic field, with a uniform $p_{x}+i p_{y}$ pairing order parameter, which is a non-Abelian topological phase with chiral Majorana and charge modes at the boundary. Recent experiments suggest the existence of a proximate nematic phase at $\nu=5 / 2$. This finding motivates us to consider an inhomogeneous paired state- $\mathrm{a} p_{x}+i p_{y}$ pair-density wave (PDW) - whose melting could be the origin of the observed liquid-crystalline phases. This state can viewed as an array of domain and antidomain walls of the $p_{x}+i p_{y}$ order parameter. We show that the nodes of the PDW order parameter, the location of the domain walls (and antidomain walls) where the order parameter changes sign, support a pair of symmetry-protected counterpropagating Majorana modes. The coupling behavior of the domain-wall Majorana modes crucially depends on the interplay of the Fermi energy $E_{F}$ and the PDW pairing energy $E_{\mathrm{PDW}}$. The analysis of this interplay yields a rich set of topological states: (1) In the weak-coupling regime $\left(E_{F}>E_{\mathrm{PDW}}\right)$, the hybridization of domain walls leads to a Majorana Fermi surface (MFS), which is protected by inversion and particle-hole symmetries. (2) As the MFS shrinks towards degenerate Dirac points, lattice effects render it unstable towards an Abelian stripe phase with two copropagating Majorana modes at the boundary. (3) A uniform component of the order parameter, which breaks inversion symmetry, gaps the MFS and causes the system to enter a non-Abelian $\mathrm{FQH}$ state supporting a chiral Majorana edge state. (4) In the strong-coupling regime, $E_{F}<E_{\mathrm{PDW}}$, the bulk fermionic spectrum becomes gapped; this is a trivial phase with no chiral Majorana edge states, which is in the universality class of an Abelian Halperin paired state. The pair-density-wave order state in a paired FQH system provides a fertile setting to study Abelian and non-Abelian FQH phases-as well as transitions thereof - tuned by the strength of the paired liquid crystalline order.
\end{abstract}

\section{INTRODUCTION}

Fractional quantum Hall (FQH) states are the quintessential example of topological electronic systems. While the majority of the $\mathrm{FQH}$ plateaus are observed near filling fractions $\nu=p / q$ with odd denominators [1], evendenominator $\mathrm{FQH}$ states $[2,3]$ provide a fertile arena to study exotic non-Abelian statistics [4,5], as well as the interplay between symmetry breaking and topological orders.

In addition to $\mathrm{FQH}$ states, a host of symmetry-breaking states have also been observed in two-dimensional electron

\footnotetext{
*These authors contributed equally to this work.
}

Published by the American Physical Society under the terms of the Creative Commons Attribution 4.0 International license. Further distribution of this work must maintain attribution to the author(s) and the published article's title, journal citation, and DOI. gases (2DEGs) in magnetic field in various Landau levels (LL). These states, generally known as electronic liquid crystal phases [6,7], break spatial symmetries to various degrees. Examples of such states are crystals (Wigner crystals [1] and bubble phases [8]), stripe phases $[7,9,10]$, and electronic nematic states [7,11]. While crystal phases break translation and rotational invariance (down to the point group symmetry of the underlying lattice), stripe (or smectic) phases break translation invariance along one direction (and concomitantly rotation symmetry), and nematic phases only break rotational invariance and are spatially uniform [12]. Most of the stripe and nematic phases that have been seen so far in experiments are compressible and do not exhibit the (integer or fractional) quantum Hall effect, although they occur in close proximity to such incompressible states. Compressible nematic phases exhibit strong transport anisotropies, which is how they are detected experimentally. In addition, stripe 
phases also exhibit strong pinning and nonlinear transport at low bias. Compressible electronic nematic order was first observed at filling fractions in $N \geq 2$ LL such as $\nu=9 / 2$, $11 / 2$, etc., [13-15]. Evidence for transition from a stripe to a nematic order in the $N=2 \mathrm{LL}$ in a compressible regime has also been seen quite recently [16].

On the other hand, in the $N=1 \mathrm{LL}, \mathrm{FQH}$ states observed [2] at $\nu=5 / 2$ are presumably paired states of the Moore-Read type [4]. In addition, other paired FQH states have been proposed to explain the plateau observed at $\nu=5 / 2$ [17-21]. Remarkably, experimental results in the $N=1$ LL also show the existence of states with nematic order, originally in samples where rotation symmetry is explicitly broken by an in-plane magnetic field [22-27]. More recently, a spontaneously formed nematic phase has been reported in GaAs/AlGaAs samples under hydrostatic pressure [28]. (See also Refs. [29,30].) The mechanism behind this spontaneous nematicity remains an open problem, and it has been speculated that it could be due to a Pomeranchuk instability of the composite fermions, as indicated by a recent numerical calculation [31]. In all of these experiments, the nematic phase is compressible, it arises after the gap or the $5 / 2 \mathrm{FQH}$ state has vanished, and it does not have a quantum Hall plateau. Magnetoresistance measurements show that the isotropic $5 / 2 \mathrm{FQH}$ state collapses at a hydrostatic pressure $P_{c} \approx 7.8 \mathrm{kbar}$. This collapse is followed by the onset of a compressible nematic state detected as a strong and temperature-dependent longitudinal transport anisotropy at higher pressures. This nematic phase persists up to a critical value of $10 \mathrm{kbar}$, where the $2 \mathrm{DEG}$ appears to become a Fermi liquid.

In addition, experiments also discovered, in the $N=1$ LL, a large nematic susceptibility (with a strong temperature dependence) in the FQH state with $\nu=7 / 3$ [32]. This experimental finding suggests that, at least in the $N=1$ Landau level, nematic and/or stripe order may also occur in proximity and/or coexistence with a FQH topological state.

The experimental observation of (presumably) paired $\mathrm{FQH}$ states in close proximity to nematic, and possibly stripe, phases suggests that all of these phases may have a common physical origin and that these orders may actually be intertwined rather than simply competing with each other. This scenario is strongly reminiscent of the current situation in cuprate superconductors and other strongly correlated oxides, where superconducting orders are intertwined, rather than competing, with stripe or nematic phases $[33,34]$. The prototype of an intertwined superconducting state is a superconducting state known as the pair-density wave (PDW) [35]. The PDW is a paired state that spontaneously breaks translation invariance. Its order parameter is closely related to that of the LarkinOvchinnikov state (although occurring in the absence of a Zeeman coupling to an external magnetic field).
A system of electrons in a half-filled Landau level, such as the $N=1 \mathrm{LL}$ in the case of the $5 / 2 \mathrm{FQH}$ state, is equivalent to a system of composite fermions [36,37] coupled to a Chern-Simons gauge field, in which two flux quanta have been attached to each electron [38]. The composite fermions are coupled to both the external magnetic field and to the dynamical Chern-Simons gauge field. In a half-filled Landau level, the composite fermions experience, on average, an effective zero magnetic field. The resulting (mean-field state) forms a Fermi surface (FS) of composite fermions [39]. In this representation, the topological incompressible isotropic $\mathrm{FQH}$ at $\nu=5 / 2$ arises from a pairing instability of the composite fermion Fermi surface, resulting in a chiral paired state. In other words, the paired $\mathrm{FQH}$ state can be viewed as a superconductor with $p_{x}+i p_{y}$ pairing coupled to a dynamical Chern-Simons gauge field at level 2 .

The aim of this paper is to construct an intertwinedorders scenario for a $2 \mathrm{DEG}$ proximate to a paired MooreRead state [4] near the $\nu=5 / 2$ filling fraction. The state that we propose is a stripe state that locally has a $p_{x}+i p_{y}$ form while, at the same time, breaking unidirectional translation invariance. We call the resulting intertwined state a $p_{x}+i p_{y}$ paired density wave state (instead of the $d$-wave local pairing of the PDW state of the cuprate high- $T_{c}$ superconductors). Such a state may also occur as an inhomogeneous version of a topological $p_{x}+i p_{y}$ superconductor as well. Here, we do not consider other types of paired states $[17,18,20]$. Nontranslational-invariant states with local $p_{x} \pm i p_{y}$ pairing were considered recently as a possible way to restore the Landau-level particle-hole symmetry (broken by both the Pfaffian and the anti-Pfaffian paired states) [40], as well as a paired and particle-hole symmetric state driven by disorder [19]. These states have different physical properties than those of the $p_{x}+i p_{y}$ PDW state we present in this paper.

In this work, we formulate a theory of a $p_{x}+i p_{y}$ PDW state, which is an interesting superconducting state in its own right, and later examine the resulting $\mathrm{FQH}$ state by considering the effects of coupling this PDW state to the dynamical Chern-Simons gauge field. The resulting state has the remarkable property of having neutral fermionic excitations that are either gapless or gapped (with nontrivial band topology). In the gapless case, the neutral (Majorana) fermions form Fermi surfaces and, hence, have a finite bulk thermal conductivity. At the same time, this state is incompressible in the charge channel; it has a plateau with a precisely defined Hall conductivity and protected chiral charge edge states.

The $p_{x}+i p_{y}$ PDW FQH state can be viewed as an array of stripes of Moore-Read states in which the $p_{x}+i p_{y}$ pair field changes sign from one stripe to the next, in close analogy to the (time-reversal-invariant) PDW superconductor discussed in the context of high-temperature superconductors [33]. This unidirectional state breaks translation 
invariance along one direction and also breaks rotations by $90^{\circ}$. Since, locally, it is equivalent to a Moore-Read state, this state also breaks the particle-hole symmetry of the Landau level. The $p_{x}+i p_{y}$ PDW FQH state can arise either by spontaneous symmetry breaking of translation (and rotation) symmetry, by the explicit breaking of rotation symmetry by a tilted magnetic field, or by inplane strain, as in the very recent experiments by Hossain and co-workers [41]. The $p_{x}+i p_{y}$ PDW FQH breaks timereversal symmetry as much as the uniform $p_{x}+i p_{y}$ paired state does. In contrast, the particle-hole symmetric stripe Pfaffian state proposed by Wan and Yang [40] consists of an array of alternating $p_{x}+i p_{y}$ and $p_{x}-i p_{y}$ stripes. While both states break translation (and rotation) symmetry, the $p_{x}+i p_{y}$ PDW FQH state breaks the Landau-level particlehole symmetry, whereas the Wan-Yang state does not. Thus, the $p_{x}+i p_{y}$ PDW breaks time-reversal invariance explicitly, whereas in the Wan-Yang state, time-reversal symmetry is equivalent to a translation by half the period of the state. These differences lead to profound differences in their spectra and physical properties.

How is the $p_{x}+i p_{y}$ PDW state related to the observed phenomena in the $N=1$ Landau level? The physical picture that we propose is that this competitor of the uniform paired state may be responsible for the observed complexity of the phase diagram in these 2DEGs. To be more specific, upon quantum and/or thermal melting, this state may give rise to a sequence of phase transitions, much in the same way as envisioned in Ref. [7]. Thus, upon melting the stripe order, a nematic state may ensue that coexists with the FQH state, similar to what was found in the experiments of Xia and co-workers [32] in the $\nu=7 / 3$ plateau, or in a compressible regime, as in the pressuredriven experiments of Samkharadze and co-workers [28] in the regime with a $\nu=5 / 2$ filling fraction. From this perspective, the nematic order would not be a "primitive" order but a "vestigial" one. An example of such a phase diagram was studied in the context of the (proposed) PDW superconducting state for the cuprates [42]. In this sense, we can think of the compressible nematic state observed in Ref. [28] as a form of vestigial order of a putative $p_{x}+i p_{y}$ PDW paired state. We note that theories of Laughlin FQH states that coexist with nematic order have also been discussed in the literature [43-49].

There has been much work on the role of particle-hole symmetry vis-à-vis the paired FQH states. The Moore-Read Pfaffian state [4] (and its anti-Pfaffian cousin [17,18]) is not particle-hole symmetric. The $p_{x}+i p_{y}$ PDW paired state presented here is based on the Moore-Read state and, as such, is not particle-hole symmetric either. Several particlehole symmetric states have been proposed. One such state is the PH-Pfaffian state [50]. Given the strong numerical evidence for both Pfaffian and anti-Pfaffian states, inhomogeneous states that restore particle-hole symmetry on large scales, while breaking it at the local level, have been proposed. Two of these proposals advocate disorder as the driving mechanism [19,21], while another one [40] proposes a stripe-type state, with alternating Pfaffian and antiPfaffian stripes. One feature of both the disorder-driven states and the PH symmetric stripe state is a nonquantized thermal Hall conductivity coexisting with a quantized Hall conductivity. This feature is also present in the $p_{x}+i p_{y}$ PDW paired state but for a different reason: It harbors bulk (anisotropic) Fermi surfaces of Bogoliubov (Majorana) quasiparticles while supporting a chiral charge edge mode as in any $\mathrm{FQH}$ state.

Regarding the fluctuations of the PDW phase, because the PDW order parameter couples to fermions bilinearly, the fluctuations of the amplitude of the PDW order will mediate interaction effects of the Majorana modes. If such effects are weak, we expect the resulting interacting state to be similar to a Fermi liquid, since it is only electrically neutral, albeit with possible changes in the Majorana bare velocities due to coupling with bosonic modes [51]. Thus, the Majorana FS should remain robust if the amplitude fluctuations are sufficiently weak.

The $p_{x}+i p_{y}$ PDW paired state is a two-dimensional state with spontaneously broken translation symmetry. As such, it is fragile to the effects of thermal fluctuations and disorder. In a strictly continuum system, dislocations of the broken translation symmetry cost a finite amount of energy, which causes the state (a smectic) to melt at any finite temperature, resulting in a state with broken orientational invariance, a nematic [52]. This feature is also present in PDW superconductors [53]. However, coupling to an underlying lattice (however weakly, and provided the stripe order is incommensurate) makes the energy of a dislocation logarithmically divergent, leading to a Kosterlitz-Thouless transition where the translation symmetry is fully restored. However, in this scenario, the orientational order is more robust since the associated symmetry is the point group symmetry of the lattice, a square lattice in the case of GaAs 2DEGs.

On the other hand, disorder has much more serious effects on states that break translation invariance, such as the $p_{x}+i p_{y}$ PDW paired state. Strictly speaking, in two dimensions, there is no true long-range order of this type for any amount of disorder [54], although the length scale over which disorder effects become prevalent may be exponentially long in a very clean system. In addition, disorder also couples to the nonchiral Majorana states bound to the domain walls we used to construct the $p_{x}+i p_{y}$ PDW paired state, by breaking inversion symmetry locally. This breaking results in a local, random, Majorana mass term for the states of each wall. This type of disorder does not lead to an Anderson insulator. Instead, for a single isolated wall, this system is equivalent to a critical transverse-field Ising model with quenched disorder [55-57], which is known to be described by an infinite disorder fixed point [58], in which the system remains critical. While this is true for an isolated wall, the behavior 
of a collection of such walls, coupled by tunneling of the Majorana fermions, has not been investigated and remains an open problem. It is quite possible that the bulk 2D state may still be a thermal metal of sorts.

This work is organized as follows. In Sec. II, we set up the proposed $p_{x}+i p_{y}$ PDW state and present a summary of the main results, both as a possible superconducting state and as an inhomogeneous paired FQH state. In Sec. III, we present a theory of the $p_{x}+i p_{y}$ paired state. Here, we present the solution of the Bogoliubov-de Gennes (BdG) equations for this PDW state and discuss in detail the properties of its fermionic spectrum. In Sec. IV, we study the coexistence of the PDW order and the uniform pairing order. In Sec. V, we use this construction to infer the properties of the $p_{x}+i p_{y}$ PDW FQH state. Section VI is devoted to the experimental implications of this PDW state and to conclusions. Theoretical details are presented in the Appendices A, B, C, and D.

\section{THE $p_{x}+i p_{y}$ PAIR-DENSITY WAVE: SETUP AND RESULTS}

In this section, we present a summary of the $p_{x}+i p_{y}$ PDW state. The pairing order parameter of the uniform $p_{x}+i p_{y}$ state has the form $\Delta(\boldsymbol{p})=\Delta\left(p_{x}+i p_{y}\right)$ (with $\Delta=$ constant). Its effective $\mathrm{BdG}$ Hamiltonian is $H=$ $\sum_{p}\left[\left(\boldsymbol{p}^{2} / 2 m\right)-\mu\right] \psi_{p}^{\dagger} \psi_{p}+\Delta(\boldsymbol{p}) \psi_{-p} \psi_{p}+$ H.c., where $m$ is the composite fermion effective mass and $\mu$ is the chemical potential [59]. In the "weak-pairing phase" of Ref. [59], where $\mu>0$, this system is a chiral topological superconductor, where all bulk fermionic excitations are gapped and there is a chiral Majorana edge state propagating along the boundary separating the topological $p$-wave state and the vacuum.

The $p_{x}+i p_{y}$ PDW state that we propose here is a version of this state with a spatially modulated order parameter of the form $\Delta \sim \Delta_{\mathrm{PDW}} f(\boldsymbol{Q} \cdot \boldsymbol{r})$, where $f$ is a periodic function with period $\lambda=2 \pi / Q$, such that the nodes of $f$ correspond to domain walls (DWs) and antidomain walls (ADWs), where the order parameter is suppressed, thus allowing for the existence of low-energy modes localized on these nodes. Here, for simplicity, we consider only unidirectional order.

In the language of superconductors, our theory is analogous to the PDW state conjectured for the cuprates whose order parameter has wave vector $\boldsymbol{Q}=(Q, 0)$ and that locally has a $d$-wave superconducting (SC) order parameter [35,6064]. The main difference is that the PDW state that we consider here has, instead, local $p_{x}+i p_{y}$ pairing order. Although at the level of the Landau-Ginzburg theory the $d$ PDW and the $p_{x}+i p_{y}$-PDW are virtually identical, their fermionic spectra are drastically different as are their topological properties.

Before moving forward with our analysis of this problem, we stress important differences between the low-energy fermion states we encounter in this work, which are associated with the spatial modulation of the PDW order parameter, and those discussed by Read and Green [59]. As discussed in Ref. [59], the edge state of the $p_{x}+i p_{y}$ paired state is a chiral Majorana fermion theory. The existence of this chiral branch is of topological origin since the edge represents a Chern number changing transition from $\mathcal{C}=1$ (in the bulk of the paired state) to $\mathcal{C}=0$ (in vacuum). This change in the Chern number is also tied to the change in the sign of the chemical potential in the BdG Hamiltonian because the region with $\mu>0$ is topological $(\mathcal{C}=1)$ and that with $\mu<0$ is trivial $(\mathcal{C}=0)$; as such, it is identified with the vacuum state.

In our analysis of the bulk properties of the PDW state, we are always in the regime where $\mu>0$ (and constant) throughout the system, and we consider the effects of a change in the overall sign of the $p_{x}+i p_{y}$ order parameter. In this stripe system, regions where the order parameter is nonzero (regardless of whether it is positive or negative) have the same Chern number $\mathcal{C}=1$. In spite of this, we demonstrate that the nodes of the order parameter still support gapless modes. Instead of a single chiral Majorana branch as in the edge of the system discussed in Ref. [59], a node of the PDW order parameter supports two nonchiral Majorana branches.

Below, we show that the Lagrangian of the effective lowenergy theory at each isolated domain wall is

$$
\mathcal{L}_{\mathrm{DW}}=i \psi_{R}\left(\partial_{t}-v \partial_{y}\right) \psi_{R}+i \psi_{L}\left(\partial_{t}+v \partial_{y}\right) \psi_{L},
$$

where $\psi_{L / R}$ represent left/right-moving massless Majorana fermions. This pair of neutral fermion modes-whose spectrum is identical to that of the one-dimensional critical quantum Ising model - owe their existence to a combination of mirror and chiral (in class BDI [72]) symmetries inherent to the Larkin-Ovchinnikov order parameter, as well as to the $p_{x}+i p_{y}$ character of the order parameter. In fact, the chiral $p$-wave nature of the order parameter plays a crucial role in the stability of the fermion zero modes on the nodes of the order parameter. See Ref. [65] for an earlier analysis similar in spirit to ours but in a rather different context of finite-momentum $s$-wave superfluids produced by imbalanced cold Fermi gases; it has found Caroli-De Gennes-Matricon midgap states supported at an isolated node of the $s$-wave order parameter, in contrast to the Majorana zero modes of the $p_{x}+i p_{y}$ PDW state.

We further show that the coupling between the domainwall counterpropagating Majorana modes leads to a highly nontrivial fermionic spectrum. In general, the (Majorana) fermionic excitations remain gapless. Their energy bands cross at the Fermi level, leading to a twofold degenerate Majorana Fermi surface. The Majorana Fermi surface is of topological origin, and the band crossing is protected by a combination of particle-hole symmetry and inversion symmetry [66]. Again, the inversion symmetry here crucially 


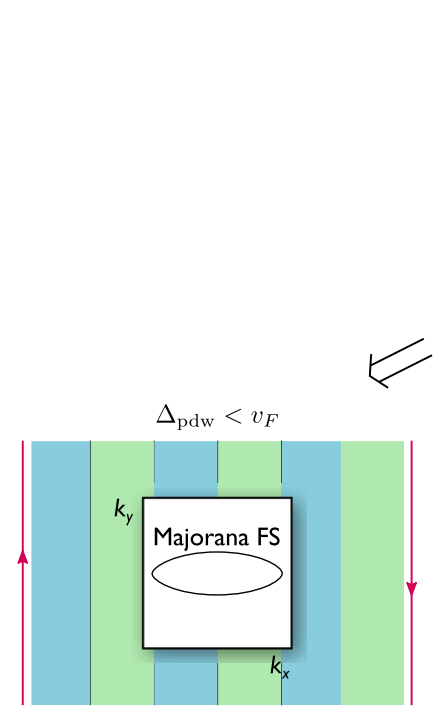

(b)
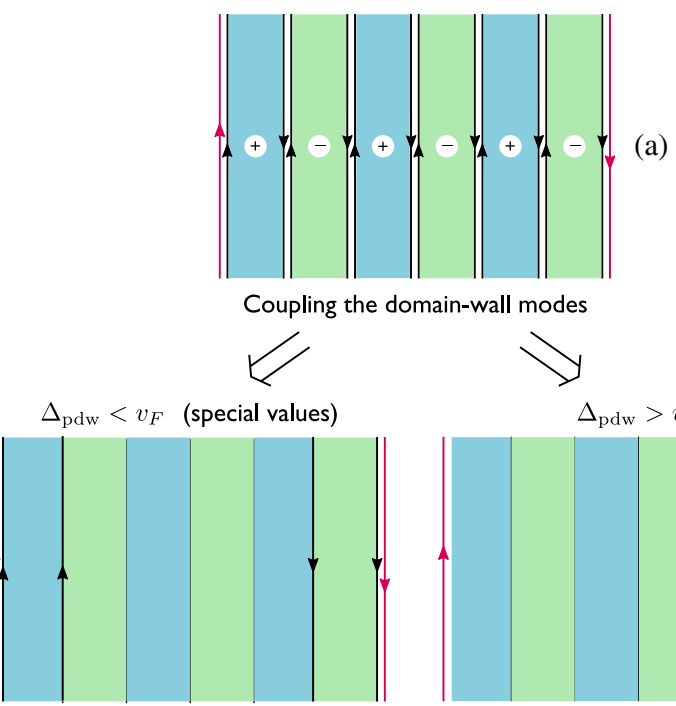

(c)

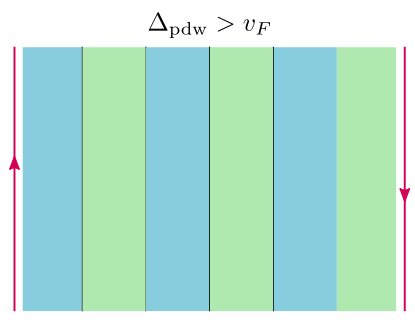

(d)

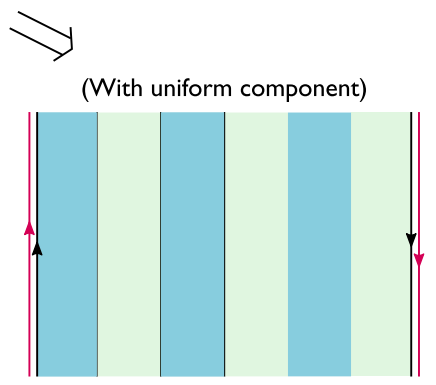

(e)

FIG. 1. Illustration of the different quantum Hall states from the stripe pairing order. The blue and green strips denote regions with positive and negative local pairing order parameters. The black arrows denote chiral Majorana modes, while the red arrows denote chiral bosonic charge modes. Panel (a): Gapless modes on domain walls of the PDW and on physical edges in the limit of vanishingly small localization length and negligible couplings between these modes. Panel (b): For the PDW order parameter $\Delta_{\mathrm{PDW}}<v_{F}$, in general, the domain-wall modes form a Majorana FS, while there is an energy gap for charge excitations. Panel (c): For particular values (see Sec. III D 1) of PDW order parameter $\Delta_{\mathrm{PDW}}<v_{F}$, the Majorana FS shrinks to zero and the fermionic sector becomes gapped. In our model, this phase has $\mathcal{C}=2$ and Abelian topological order. Panel (d): For $\Delta_{\mathrm{PDW}}>v_{F}$, the fermionic sector becomes trivially gapped, and the resulting quantum Hall state is an Abelian one. Panel (e): The neutral FS at $\Delta_{\mathrm{PDW}}<v_{F}$ becomes gapped with a uniform $p_{x}+i p_{y}$ pairing component with nontrivial topology. The resulting quantum Hall state has non-Abelian topological order just like the Pfaffian state.

relies on both the $p$-wave character of the local pairing and the Larkin-Ovchinnikov order parameter. For PDW states, in general, one expects a gapless fermionic spectrum, as a weak PDW order parameter opens gaps only at selected points in $k$ space. In those cases, the excitations form a Bogoliubov Fermi surface (pocket), which is closely tied to the original normal-state Fermi surface. Along the Bogoliubov Fermi surface, the quasiparticles alternate from being more electronlike to more holelike. Here, we stress that the Majorana Fermi surface is distinct from the original normal-state Fermi surface, and it satisfies the Majorana condition $\gamma^{\dagger}(-\boldsymbol{k})=$ $\gamma(\boldsymbol{k})$ everywhere. Moreover, in particular ranges of the PDW order parameter, the fermionic spectrum becomes gapped. Interestingly, the topology of these gapped phases is distinct from a uniform $p_{x}+i p_{y}$ state with a Chern number $\mathcal{C}=1$. Instead, we find phases with both $\mathcal{C}=2$ and $\mathcal{C}=0$, even though locally the pairing is identical to a $p_{x}+i p_{y}$ pairing state.

The bulk regions where $\Delta(\boldsymbol{r})$ is nonzero (which is everywhere except on isolated one-dimensional lines extended in the $y$ direction) have the same Chern number and the same Hall response, irrespective of the overall sign of the order parameter. Consequently, the system is a quantum Hall insulator with respect to the charge modes (albeit with a spatial-dependent charge gap) while supporting low-energy excitations in the form of gapless neutral fermions supported along the domain walls. Thus, while
Majorana fermions may tunnel as soft excitations on the PDW domain walls, electron tunneling is suppressed everywhere in the bulk (including along the domain walls) due to the charge gap. The resulting state is an exotic heat conductor but an electric insulator.

Our detailed investigation of the properties of fermionic excitations of the $p_{x}+i p_{y}$ PDW state finds that this system represents a symmetry-protected topological phase whose remarkably rich properties are summarized as follows:

(1) Each isolated DW supports a pair of massless Majorana fermions, as shown in Fig. 1(a), which are protected by the unitary symmetry $\mathcal{U}=\mathcal{M}_{y} \mathcal{S}$, where $\mathcal{M}_{y}$ is the mirror symmetry along the direction of the domain wall and $\mathcal{S}$ is a chiral symmetry (in class BDI). In the presence of a uniform component $\Delta_{\mathrm{u}}$ of the $p_{x}+i p_{y}$-wave order parameter that preserves $\mathcal{U}$ symmetry, the massless Majorana fermions cannot be gapped out for $\left|\Delta_{\mathrm{u}}\right|<\left|\Delta_{\text {PDW }}\right|$, whereas no massless Majorana fermions exist in DWs for $\left|\Delta_{\mathrm{u}}\right|>\left|\Delta_{\text {PDW }}\right|$, representing the phase adiabatically connected to the uniform $p_{x}+i p_{y^{-}}$ wave state [59].

(2) For $\Delta_{\mathrm{PDW}}<v_{F}$, where $v_{F}$ is the Fermi velocity of the composite Fermi liquid, there exists, in general, a twofold degenerate Majorana Fermi surface (made out of Majorana fermions), protected by the particlehole symmetry and the inversion symmetry of the 
PDW state. As stated above, this state supports gapless neutral excitations but is an electric insulator. This state is one of the main findings of the present work, and we illustrate this phase in Fig. 1(b).

As $\Delta_{\text {PDW }}$ varies, this Majorana Fermi surface shrinks and expands periodically, and when the Majorana Fermi surface shrinks to zero, the fermionic spectrum becomes gapped. We find that this gapped state has a Chern number $\mathcal{C}=2$ even if the local pairing is of $p_{x}+i p_{y}$ form. This result can be understood as the result of a Chern-number-one contribution from the bulk $p_{x}+i p_{y}$ pairing order in addition to a Chern-number-one contribution from the domain walls. The corresponding quantum Hall state has Abelian topological order, as the vortices of the pairing order do not host Majorana zero modes. The edge conformal field theory (CFT) consists of a charge mode and two Majorana fermions; in total, it has a chiral central charge $c=2$. This phase is illustrated in Fig. 1(c).

(3) For PDW states with $\Delta_{\text {PDW }}>v_{F}$, the fermionic spectrum is gapped [see Fig. 1(d)]. From the fermionic point of view, this gapped phase is topologically trivial with $\mathcal{C}=0$ as it does not support chiral edge Majorana fermions. In the $\mathrm{QH}$ setting, we identify this phase with the stripe Halperin Abelian quantum Hall state where electrons form tightly bound charge- $2 e$ bosons that condense in a stripe Laughlin state.

(4) The bulk spectrum changes in the presence of a uniform component $\Delta_{\mathrm{u}}$ of the $p_{x}+i p_{y}$ pairing order. For $\Delta_{\mathrm{PDW}}<v_{F}$, the Majorana FS becomes gapped by an infinitesimal $\Delta_{\mathrm{PDW}}$, while for $\Delta_{\text {PDW }}>v_{F}$, the trivial gapped phase survives until a critical value of $\Delta_{\mathrm{u}}$. We find that the gapped phase with $\Delta_{\mathrm{u}}$ has a Chern number $\mathcal{C}=1$, i.e., is in the same phase as the uniform Moore-Read $p_{x}+i p_{y}$ state. This phase is represented in Fig. 1(e). Thus, interestingly, the neutral FS in Fig. 1(b) represents a quantum critical "phase" that separates distinct neutral fermion edge states.

Based on our detailed analysis in the remainder of the paper, all of these phases mentioned above have been placed in a schematic mean-field phase diagram, shown in Fig. 10.

\section{FERMIONIC SPECTRUM OF THE $p_{x}+i p_{y}$ PAIR-DENSITY WAVE}

The quantum Hall state with a half-filled Landau level can be viewed as the paired state of the composite fermions coupled to both a dynamical gauge field and the external electromagnetic field. In this section, we analyze the spectrum of the fermionic sector described by the mean-field pairing of composite fermions. We postpone a full description of the quantum Hall state with gauge fields and charge modes to Sec. V.

The analysis in this section also serves as a selfcontained mean-field theory for the $p_{x}+i p_{y}$ PDW superconductor, which could potentially be relevant for, e.g., $\mathrm{Sr}_{2} \mathrm{RuO}_{4}$ [67] or superfluid ${ }^{3} \mathrm{He}$ [68]. To our knowledge, this theory has not been presented before in the literature.

\section{A. BdG description of the $\boldsymbol{p}_{\boldsymbol{x}}+\boldsymbol{i} \boldsymbol{p}_{\boldsymbol{y}}$ PDW state}

Before turning to a PDW state, we consider a generic two-dimensional state with $p_{x}+i p_{y}$ local pairing symmetry. We begin with the BdG Hamiltonian in the continuum

$$
H(\boldsymbol{r})=\left(\begin{array}{cc}
\epsilon(\boldsymbol{k}) & \frac{1}{2}\left\{k_{-}, \Delta(\boldsymbol{r})\right\} \\
\frac{1}{2}\left\{k_{+}, \Delta^{*}(\boldsymbol{r})\right\} & -\epsilon(\boldsymbol{k})
\end{array}\right),
$$

where $\boldsymbol{k}=\left(k_{x}, k_{y}\right)=\left(-i \partial_{x},-i \partial_{y}\right), \quad k_{ \pm}=k_{x} \pm i k_{y}=-i \partial_{ \pm}$ (we set $\hbar=1$ ). For now, let us take the simplest Galileaninvariant continuum dispersion

$$
\epsilon(\boldsymbol{k})=\frac{\boldsymbol{k}^{2}}{2 m}-\mu .
$$

We later discuss the lattice effects of the $\mathrm{BdG}$ Hamiltonian. Here, the anticommutator $\left\{k_{-}, \Delta(\boldsymbol{r})\right\} \equiv$ $k_{-} \Delta(\boldsymbol{r})+\Delta(\boldsymbol{r}) k_{-}$is taken to symmetrize the $\boldsymbol{r}$ dependence and $\boldsymbol{p}$ dependence, a standard procedure to treat a nonuniform order parameter $\Delta(\boldsymbol{r})$.

Throughout this work, we consider the case with a normal-state FS, i.e., $\mu>0$, which, in the case of a uniform order parameter $\Delta$, corresponds to the weak-pairing regime, describing a topological paired state with chiral Majorana fermion edge states [59]. Notice that the name strongpairing regime has been used by Read and Green [59] for cases with $\mu<0$. Even though we consider cases with a large pairing order $|\Delta|$, it should not be confused with the strong-pairing regime in the sense of Read and Green.

The BdG Hamiltonian of Eq. (3.1) possesses a particlehole symmetry

$$
\sigma_{1} H \sigma_{1}=-H^{*},
$$

which relates positive and negative energy states: If $\Psi_{E}(\boldsymbol{r})=\left\langle\boldsymbol{r} \mid \Psi_{E}\right\rangle$ is an eigenmode of $H$ with energy $E$, then $\sigma_{1} \Psi_{E}^{*}$ is an eigenmode with energy $-E$. Of these states, a particularly interesting eigenstate is the zero mode, with $E=0$. It satisfies $\sigma_{1} \Psi_{0}^{*}= \pm \Psi_{0}$ such that they can be expressed as $\Psi_{0}(x)=e^{-i \pi / 4} \psi(x)(1, \pm i)^{T}$, with $\psi(x) \in \mathbb{R}$.

For a PDW, the order parameter varies along the $x$ axis, $\Delta(x)$, and we work in the gauge where it is a real function of $x$. With the ansatz that the zero modes are translation invariant along the $y$ direction $\left(k_{y}=0\right)$, the equation for the potential zero modes reads 


$$
\left(-\frac{\partial_{x}^{2}}{2 m}-\mu\right) \psi(x) \pm \frac{1}{2}\left\{\partial_{x}, \Delta(x)\right\} \psi(x)=0 .
$$

It should be emphasized that these states are zero modes of the BdG Hamiltonian, and, as a result, they obey the Majorana condition. However, we see in Sec. III B that these are not isolated states in the spectrum but are actually part of a branch of propagating massless Majorana fermions, propagating along the domain wall. Thus, they should not be confused with their formal cousins, the isolated zero modes at end points of one-dimensional $p$-wave superconductors [69], or at the core of vortices of $2 \mathrm{D}$ chiral superconductors [70]. The latter type is associated with the non-Abelian statistics of these defects, whereas the massless Majorana fermions we find here are bound states of domain walls and are not associated with non-Abelian statics. For these reasons, and to avoid confusion, we do not refer to the zero modes of the BdG Hamiltonian for domain walls as Majorana zero modes.

\section{B. Domain-wall bound states}

A PDW state is characterized by pairing order parameters $\Delta_{ \pm \boldsymbol{Q}}$ (and their higher harmonics such as $\Delta_{ \pm 3 \boldsymbol{Q}}$, $\left.\Delta_{ \pm 5 \boldsymbol{Q}}, \ldots\right)$ with nonzero momentum $\pm \boldsymbol{Q}$, which couple to fermions via

$$
\begin{aligned}
H_{\mathrm{PDW}}= & \sum_{\boldsymbol{k}, \pm, n=\mathrm{odd}} \Delta_{ \pm n \boldsymbol{Q}} f_{n}(\boldsymbol{k}) \\
& \times c^{\dagger}(\boldsymbol{k} \pm n \boldsymbol{Q} / 2) c^{\dagger}(-\boldsymbol{k} \pm n \boldsymbol{Q} / 2)+\text { H.c. },
\end{aligned}
$$

where $c^{\dagger}(\boldsymbol{k})$ is a spinless fermion creation operator at momentum $\boldsymbol{k}$, and $f(\boldsymbol{k})$ is the PDW form factor that is an odd function enforced by fermionic statistics. At the level of mean-field theory, the PDW order parameters $\Delta_{ \pm \boldsymbol{Q}}$ satisfy

$$
\left|\Delta_{Q}\right|=\left|\Delta_{-Q}\right|,
$$

and this relation holds similarly for all higher harmonics. Then, the real-space form of the order parameter is

$$
\Delta(\boldsymbol{r})=\sum_{n>0}\left|\Delta_{n} \boldsymbol{Q}\right| e^{i \theta_{Q^{n}}} \cos \left(n \boldsymbol{Q} \cdot \boldsymbol{r}+\phi_{\boldsymbol{Q}_{n}}\right) .
$$

At the mean-field level, and in the absence of topological singularities, the phases $\theta_{Q_{n}}$ and $\phi_{Q_{n}}$ can both be set to zero after a gauge transformation and a spatial translation. As we shall see later, this defining property of PDW leads to important symmetries that protect a gapless fermionic spectrum. However, fluctuations about the mean-field state do not obey these constraints. As a result, the full PDW order parameter has, in its simplest form, two complex order parameters, $\Delta_{ \pm \boldsymbol{Q}}[33,60,71]$. This complexity of the order parameter manifold has important consequences for the pathways to the quantum and/or thermal melting of this state.

In real space, a PDW state can be viewed as a periodic arrangement of domains of pairing order with alternating signs of the order parameter. Across each domain wall, the pairing gap $\Delta$ changes sign and vanishes at the domain-wall location. Thus, we expect the low-energy fermionic states to be concentrated in the close vicinity of the domain walls. For simplicity, we only consider the domain-wall states with the lowest energy. The interplay between higherenergy domain-wall states can be similarly analyzed and does not lead to any qualitative differences, as we shall see later. Moreover, it turns out that, for an isolated domain wall, the lowest-energy states have interesting topological properties.

It is convenient to consider a simple picture of a PDW whose $p_{x}+i p_{y}$ order parameter has constant magnitude but alternating signs. In this simple case, the midgap states with nonzero energies are pushed away from $E=0$, and we can study the properties of Majorana zero modes more clearly. We begin our analysis with a single isolated domain wall (DW), or antidomain wall (ADW), and use the result as a starting point to couple the bound states for a DW-ADW array. It should be noted that the zero modes that we find below arise as bound states of the BdG oneparticle Hamiltonian, much in the same way as Majorana zero modes at the end point of a $p$-wave superconductor [69] (or in the cores of a half-vortex of a $p_{x}+i p_{y}$ superconductor [70]). As we noted above, their physics is very different.

We begin with a DW configuration at $x=0$, given by

$$
\Delta_{\text {isol }}(\boldsymbol{r})=-\Delta_{\mathrm{PDW}} \operatorname{sgn}(x),
$$

where $\Delta_{\mathrm{PDW}}>0$. For convenience, we define a quantity with units of momentum

$$
q=m \Delta_{\mathrm{PDW}} .
$$

The solutions to Eqs. (3.4) and (3.16) yield a pair of normalizable zero-energy solutions with $k_{y}=0$ localized at $x=0$, with even and odd parity, given by (for more details, see Appendix A)

$$
\begin{aligned}
& \left\langle\boldsymbol{r} \mid \Psi_{e}\right\rangle=\frac{N_{e}}{\sqrt{L}} e^{-q|x|} \cos (\kappa x) u_{1}, \\
& \left\langle\boldsymbol{r} \mid \Psi_{o}\right\rangle=\frac{N_{o}}{\sqrt{L}} e^{-q|x|} \sin (\kappa x) u_{1},
\end{aligned}
$$

where

$$
u_{1}=(1, i)^{T} / \sqrt{2}, \quad \kappa=\sqrt{k_{F}^{2}-q^{2}},
$$

$k_{F} \equiv \sqrt{2 m \mu}$ is the Fermi momentum, and the normalization constants $N_{e}, N_{o}$ are given by 
$N_{e}=\sqrt{\frac{2 q\left(\kappa^{2}+q^{2}\right)}{\kappa^{2}+2 q^{2}}}, \quad N_{o}=\sqrt{\frac{2 q\left(\kappa^{2}+q^{2}\right)}{\kappa^{2}}}$,

where $L$ is the system length along the $y$ direction. For $q \ll k_{F}$, we have $N_{o}=N_{e}$, but, in general, they are different.

Notice that the above expression (3.10) applies to both $q<k_{F}$ and $q>k_{F}$ : In particular, for $q>k_{F}$, the coefficient $\kappa$ is imaginary, and the $\cos (\kappa x)$ and $\sin (\kappa x)$ functions in Eq. (3.10) become $\cosh (|\kappa| x)$ and $-i \sinh (|\kappa| x)$ and are nonoscillatory. One can easily verify that the wave functions are still normalizable, thanks to the $e^{-q|x|}$ factor, with the same normalization factor $N_{e, o}$. (Note that $N_{o}$ becomes imaginary, and $\left\langle\boldsymbol{r} \mid \Psi_{o}\right\rangle$ remains real.) However, as we will see, the different forms of the wave packets for $q<k_{F}$ and $q>k_{F}$ generally lead to very different coupling between the domain-wall modes.

The dispersion relation of the propagating modes along the $y$ axis can be obtained using degenerate perturbation theory by computing the $2 \times 2$ perturbation matrix $\left[\hat{V}\left(k_{y}\right)\right]_{p, p^{\prime}}=\left\langle\Psi_{p}\left|\delta H\left(k_{y}\right)\right| \Psi_{p^{\prime}}\right\rangle$, for $p, p^{\prime}=e, o$ and

$\delta H\left(k_{y}\right)=H\left(k_{y}\right)-H\left(k_{y}=0\right)=k_{y} \Delta_{\text {isol }}(x) \sigma_{y}+\frac{k_{y}^{2}}{2 m} \sigma_{z}$.

Direct calculation gives that the eigenstates are a pair of counterpropagating modes:

$$
\left\langle\boldsymbol{r} \mid \Psi_{R, L}\left(k_{y}\right)\right\rangle=e^{i k_{y} y}\left(\left\langle\boldsymbol{r} \mid \Psi_{e}\right\rangle \mp\left\langle\boldsymbol{r} \mid \Psi_{o}\right\rangle\right) / \sqrt{2}
$$

with linear dispersion

$$
E_{R, L}= \pm v_{y} k_{y}, v_{y}=\frac{q^{2}}{m \sqrt{2 q^{2}+\kappa^{2}}} .
$$

Notice that the quadratic dependence on the momentum disappears due to $\left\langle u_{1}\left|\sigma_{z}\right| u_{1}\right\rangle=0$.

For an ADW configuration with

$$
\Delta_{\text {isol }}^{\prime}(\boldsymbol{r})=\Delta_{\mathrm{PDW}} \operatorname{sgn}(x),
$$

the counterpropagating edge states can be straightforwardly obtained by the same procedure. Since a DW and an ADW transform into each other under a gauge transformation $\Delta \rightarrow-\Delta$, much of the result above for a DW should hold for an ADW. The only difference is that the spinor part $u$ of the wave functions in Eq. (3.10) is replaced with

$$
u_{2}=(1,-i)^{T} / \sqrt{2}
$$

\section{Symmetry-protected stability of the domain-wall counterpropagating modes}

The existence of two gapless modes at the domain wall may seem surprising at first sight. After all, a domain wall separates regions with $p_{x}+i p_{y}$ pairing and $-\left(p_{x}+i p_{y}\right)$ pairing, and the two regions have the same Chern number. Thus, without additional symmetry, the domain-wall states are generally gapped.

To establish the stability of the domain-wall modes, it is convenient to "fold" the system along a single domain wall and treat the domain wall as the edge of the folded system. The symmetry that is pertinent to the stability of the edge modes involves a spinless time-reversal $\mathcal{T}=K$ operation ( $K$ is the complex conjugation operator). For a $p_{x}+i p_{y}$ state, both the (spinless) time-reversal symmetry $\mathcal{T}$ and the mirror symmetries $\mathcal{M}_{x, y}$ are broken, but one can define a composite symmetry $\mathcal{M}_{x, y} \mathcal{T}$ that remains intact. Together with the particle-hole symmetry $\mathcal{C}=\tau_{x} K$ that comes with the BdG Hamiltonian, our (folded) system has a $\mathcal{M}_{y} \mathcal{S}$ symmetry, where $\mathcal{S}=\mathcal{C} \mathcal{T}=\tau_{x}$ is known as a chiral operation [72]. The system satisfies

$$
\left(\mathcal{M}_{y} \mathcal{S}\right) \mathcal{H}\left(\mathcal{M}_{y} \mathcal{S}\right)^{-1}=-\mathcal{H}
$$

For the mirror-invariant value $k_{y}=0$, the composite symmetry reduces to a chiral symmetry $\mathcal{S}$, and the 1D subsystem belongs to the BDI class [72]. According to the classification table, the BDI class in one dimension has a $\mathbb{Z}$ classification characterized by an integer winding number $\nu$. We find that the folded system has $\nu=2$, and this corresponds to the two zero modes at the edge at $k_{y}=0$. One can show that a term of about $\Delta^{\prime} \sigma_{y}$ added to the Hamiltonian of Eq. (3.1) would gap out these two modes, but such a term is prohibited by $\mathcal{M}_{y} \mathcal{S}$ symmetry.

We note that the chiral symmetry stems from the defining symmetry of the PDW state. In general, nonuniform superconducting states consist of finite-momentum pairing order parameters $\Delta_{Q}$ and $\Delta_{-}$, which are related by inversion. The Fulde-Ferrel state, for which $\Delta_{Q} \neq 0$ and $\Delta_{-Q}=0$, has a single complex order parameter and does not oscillate in space. This SC order parameter in real space has a "spiral" pattern in phase rather than an oscillatory pattern. In these cases, the $\mathcal{M}_{y} \mathcal{T}$ symmetry is absent, as is the $\mathcal{M}_{y} \mathcal{S}$ symmetry, and there are no such gapless domainwall modes. It is crucial that, for a PDW state, similar to a Larkin-Ovchinnikov (LO) state, $\left|\Delta_{Q}\right|=\left|\Delta_{-Q}\right|$, such that the $\mathcal{M}_{y} \mathcal{S}$ symmetry is intact.

\section{FS from domain-wall coupling}

So far, we have considered the case of completely isolated DWs. At finite values of the PDW wavelength, though, hybridization between DWs inevitably occurs, and it is responsible for making the DW excitations regain their 2D character. In this subsection, we consider a PDW state 
with DW (and ADW) bound states and derive the dispersion of the (hybridized) bulk states.

Because of the exponential decay of the domain-wall state wave function in Eq. (3.10), we expect that the effective hopping matrix elements between DWs separated by distance $d$ scale as $e^{-q d}$, and, for nearest-neighbor DW and anti-DW separated by $\lambda / 2 \equiv \pi / Q$, the coupling is of the order $e^{-\pi q / Q}$. Then, if $Q<q$, we can employ a tightbinding approximation where the nearest-neighbor hopping gives the dominant contribution.

For the rest of this work, we mainly focus on the regime

$$
Q<q<k_{F},
$$

where the first inequality enables us to use a tight-binding approximation, and the second inequality ensures that the local pairing gap $\Delta(\boldsymbol{r})$ is smaller than Fermi energy $\mu$, a reasonable assumption in the spirit of the weak-coupling theory. As we discussed in Sec. III B, in this regime, the wave functions in Eq. (3.10) are oscillatory functions enveloped by symmetric exponential decay. We have set the PDW wave vector $Q<k_{F}$-this is needed in order for the normal-state FS to be reconstructed in a meaningful way. As we proceed, we discuss other regimes of the length scales with $q<Q$ and $q>k_{F}$ as well.

Consider the PDW state obtained as a periodic sequence of DWs and ADWs,

$\Delta(x)=\Delta_{\mathrm{PDW}}\left[1+\sum_{\epsilon=1,2} \sum_{n \in \mathbb{Z}}(-1)^{\epsilon} \operatorname{sgn}\left(x-x_{n}^{(\epsilon)}\right)\right]$,

where DWs are located at $x_{n}^{(1)}=n \lambda$ and ADWs are located at $x_{n}^{(2)}=(n+1 / 2) \lambda$. The order parameter of Eq. (3.20) and, consequently, the BdG Hamiltonian of the state are then periodic under shifts of $x$ by integer multiples of $\lambda$. The conclusion of this paper does not change if one uses a smooth order parameter instead of the square-wave one. The main reason is that the essential ingredients for our result are the counterpropagating Majorana modes at an isolated domain wall and the twofold degenerate Majorana FS via domain wall coupling. Both are protected by symmetry, shown in Sec. III B, and are not affected by the detailed form of the PDW order parameter. We adopt this square-wave parameter to simplify the analytical calculation.

Other than this translational symmetry, the PDW configuration (3.20) also entails an inversion symmetry of the BdG Hamiltonian (3.1) with inversion centers at $x_{n}^{(\epsilon)}$. Indeed, under such an inversion, both $k_{ \pm}$and $\Delta(\boldsymbol{r})$ change sign, rendering their anticommutator and hence $H(\boldsymbol{r})$ invariant. For the domain wall modes, from Eq. (3.14), we see that left movers and right movers transform into each other under inversion. It is straightforward to see that this inversion symmetry simply derives, from Eq. (3.6), the defining property of a PDW state.

The system also has a "half-translation" symmetry. Namely, under a translation by $\lambda / 2$, the order parameter (3.20) flips sign, but this is identical to the original state after a gauge transformation $\Delta \rightarrow-\Delta$. For the domain wall modes, left and right movers retain their chirality under the half-translation. We use these symmetries to establish relations between the hopping matrices.

Let us consider a variational state

$$
\left|\Psi_{k_{x}, k_{y}}\right\rangle=\sum_{\epsilon=1,2} \sum_{\mu=L, R} c_{\epsilon, \mu} \sum_{n \in \mathbb{Z}} \frac{e^{i k_{x} n \lambda}}{\sqrt{N}}\left|\Psi_{\epsilon, \mu, n}\left(k_{y}\right)\right\rangle,
$$

where the subscript $\epsilon=1$ denotes DW modes and $\epsilon=2$ ADW modes. Recall that $\left|\Psi_{\epsilon=1}\right\rangle \propto u_{1}=(1, i)^{T} / \sqrt{2}$ and $\left|\Psi_{\epsilon=2}\right\rangle \propto u_{2}=(1,-i)^{T} / \sqrt{2}$. The coefficients $c_{\epsilon, \mu}$ are variational parameters, the dependence on the momentum $k_{y}$ enters via the dispersive modes $\left|\Psi_{L, R}\right\rangle$ along each DW and $\mathrm{ADW}$, and the dependence on the crystal momentum $k_{x} \in$ $(-Q / 2, Q / 2)$ enforces that the state of Eq. (3.21) satisfies the Bloch theorem.

The steps leading to the energy of this variational tightbinding state are lengthy but straightforward [73], and they are presented in Appendix C. Minimization of the energy of the state

$$
E_{k_{x}, k_{y}}\left[\left\{c_{\epsilon, \mu}\right\}\right]=\frac{\left\langle\Psi_{k_{x}, k_{y}}|H| \Psi_{k_{x}, k_{y}}\right\rangle}{\left\langle\Psi_{k_{x}, k_{y}} \mid \Psi_{k_{x}, k_{y}}\right\rangle},
$$

with respect to the variational parameters $\left\{c_{\epsilon, \mu}\right\}$, yields the secular equation

$$
\operatorname{det}\left[\mathcal{H}_{k_{x}, k_{y}}-E_{k_{x}, k_{y}} \mathrm{I}_{4 \times 4}\right]=0,
$$

where the effective Hamiltonian, valid in the vicinity of $k_{y}=0$, is given by

$$
\begin{aligned}
& \mathcal{H}\left(k_{x}, k_{y}\right) \\
& =\left(\begin{array}{cccc}
v_{y} k_{y} & 0 & t^{\prime}+t^{\prime} e^{-i k_{x}} & t-\tilde{t} e^{-i k_{x}} \\
0 & -v_{y} k_{y} & -\tilde{t}+t e^{-i k_{x}} & t^{\prime}+t^{\prime} e^{-i k_{x}} \\
t^{\prime}+t^{\prime} e^{i k_{x}} & -\tilde{t}+t e^{i k_{x}} & v_{y} k_{y} & 0 \\
t-\tilde{t} e^{i k_{x}} & t^{\prime}+t^{\prime} e^{i k_{x}} & 0 & -v_{y} k_{y}
\end{array}\right),
\end{aligned}
$$

where, for convenience, we have redefined $k_{x} \lambda \rightarrow k_{x}$ so that $k_{x} \in(-\pi, \pi)$.

This effective Hamiltonian is expressed in the basis of states $\left\{\left|\Psi_{1, R}\right\rangle,\left|\Psi_{1, L}\right\rangle,\left|\Psi_{2, R}\right\rangle,\left|\Psi_{2, L}\right\rangle\right\}$ (momentum dependence omitted), where the indices 1 (2) denote DW (ADW) 


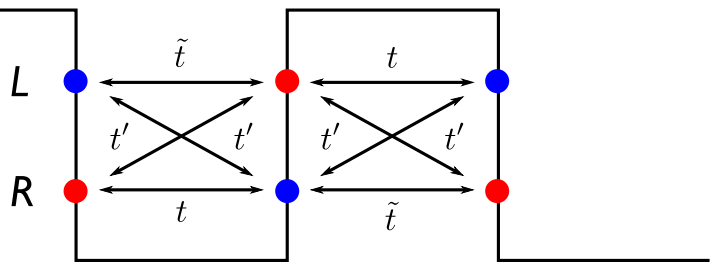

FIG. 2. The coupling $t, \tilde{t}$, and $t^{\prime}$ between the neighboring domain-wall modes.

degrees of freedom (d.o.f.). The diagonal blocks proportional to $v_{y} k_{y} \sigma_{3}$ then represent the kinetic energies of the right- and left-moving modes on DWs and ADWs, respectively, while the off-diagonal blocks represent the coupling between an adjacent DW-ADW pair. The constants $t, \tilde{t}, t^{\prime}$ can be understood intuitively as "hopping amplitudes" between the neighboring domain-wall modes, which we illustrate in Fig. 2. Specifically, $t^{\prime}$ describes the coupling between neighboring modes with the same chirality. Importantly, all of these couplings are the same following the inversion symmetry and the half-translation symmetry. Note that $t$ describes the coupling between the right mover at a DW with the left mover at an ADW to its right. By the half-translational symmetry or inversion symmetry, $t$ also describes the coupling between the right mover at an ADW with the left mover at a DW to its right. On the other hand, $\tilde{t}$ describes the coupling of a left mover with a right mover to its left. Notice that there are no symmetry requirements relating $t$ and $\tilde{t}$.

In Appendix C, we evaluate $t, \tilde{t}$, and $t^{\prime}$, and the results are

$$
\begin{aligned}
t= & -\frac{\kappa}{4 m} \exp (-q \lambda / 2) \\
& \times\left[2 N_{e} N_{o} \cos \left(\frac{\kappa \lambda}{2}\right)+\left(N_{e}^{2}-N_{o}^{2}\right) \sin \left(\frac{\kappa \lambda}{2}\right)\right], \\
\tilde{t}= & -\frac{\kappa}{4 m} \exp (-q \lambda / 2) \\
& \times\left[2 N_{e} N_{o} \cos \left(\frac{\kappa \lambda}{2}\right)-\left(N_{e}^{2}-N_{o}^{2}\right) \sin \left(\frac{\kappa \lambda}{2}\right)\right], \\
t^{\prime}= & -\frac{\kappa}{4 m} \exp (-q \lambda / 2)\left(N_{e}^{2}+N_{o}^{2}\right) \sin \left(\frac{\kappa \lambda}{2}\right) .
\end{aligned}
$$

We note that, so far, our analysis and Eqs. (3.24) and (3.25) apply to both $q<k_{F}$ and $q>k_{F}$. In particular, it is easy to verify that for $q>k_{F}, t, \tilde{t}$, and $t^{\prime}$ are still real. As promised, we focus on $q<k_{F}$ for now. In this regime, we find that, out of the four bands [74] given by Eq. (3.24), two of them cross each other at zero energy, illustrated in Fig. 3 [the eigenstate of Eq. (3.24) for $t^{\prime}=0.5, t=0.4$, $\tilde{t}=0.6$ at $\left.k_{y}=0\right]$. The zero-energy band crossing results in a (twofold degenerate) FS, whose contour is given by the vanishing of the determinant

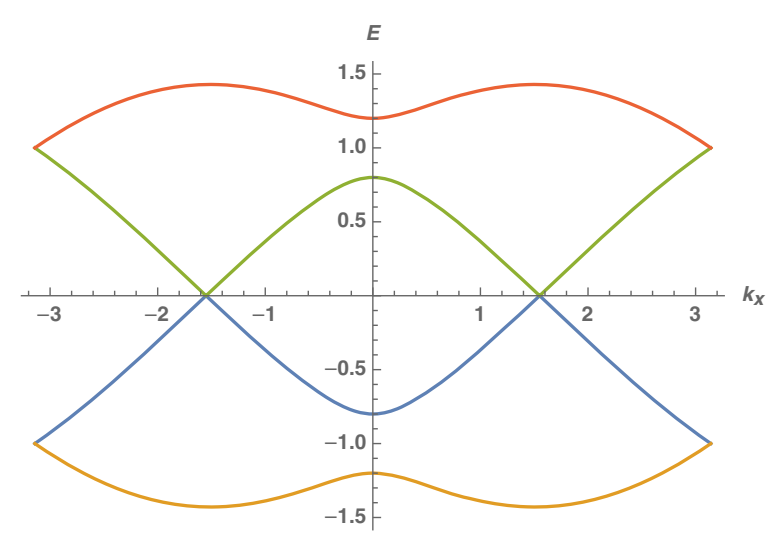

FIG. 3. The energy bands of the tight-binding Hamiltonian (3.24) for $t^{\prime}=0.5, t=0.4, \tilde{t}=0.6$ at $k_{y}=0$.

$$
\begin{aligned}
& \operatorname{det}\left[\mathcal{H}\left(k_{x}, k_{y}\right)\right] \\
& =\left[\left(v_{y} k_{y}\right)^{2}-4\left(t^{\prime 2}+t \tilde{t}\right) \cos ^{2}\left(k_{x} / 2\right)+(t+\tilde{t})^{2}\right]^{2}=0 .
\end{aligned}
$$

It is easy to verify that this equation has a solution for $q<k_{F}$. Importantly, this degenerate FS belongs to energy bands of Majorana modes, and, by construction, quasiparticles near it satisfy the Majorana condition $\gamma^{\dagger}(\boldsymbol{k})=\gamma(-\boldsymbol{k})$. For this reason, we term it a Majorana FS.

To verify Eq. (3.26), we numerically solve the lattice version of the BdG Hamiltonian (3.1). For the normal state, we use the dispersion

$$
\epsilon(\boldsymbol{k})=-t_{0}\left(\cos k_{x}+\cos k_{y}\right)-\mu,
$$

and for the off-diagonal element of the BdG Hamiltonian, we use

$$
k_{ \pm} \rightarrow \sin k_{x} \pm i \sin k_{y}
$$
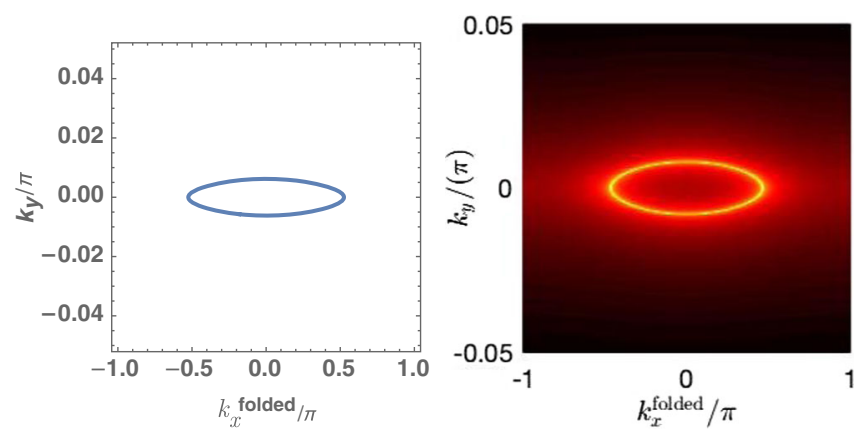

FIG. 4. FS from coupling the domain-wall modes. Left panel: FS obtained from Eq. (3.26), where the hopping parameters in Eq. (3.25) are computed for lattice parameters $t_{0}=1, \mu=-1.25$, $\Delta_{\text {PDW }}=0.82$, and $Q=\pi / 6$. Right panel: Simulated fermionic spectral function $\rho(\boldsymbol{k}, E=0)$ from a lattice nearest-neighbor hopping model subject to a PDW order parameter. 

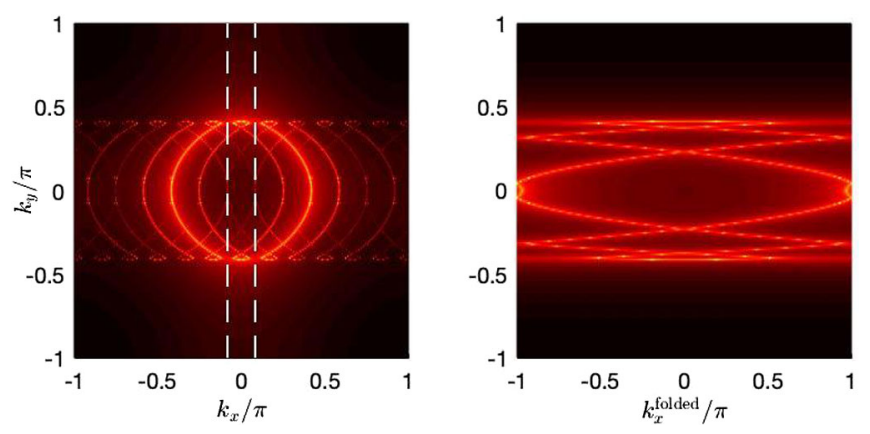

FIG. 5. Simulated fermionic spectral function $\rho(\boldsymbol{k}, E=0)$ from a lattice nearest-neighbor hopping model subject to a PDW order parameter. For the normal state, we use the dispersion $\epsilon(\boldsymbol{k})=-t_{0}\left(\cos k_{x}+\cos k_{y}\right)-\mu$, and we take the local PDW coupling with wave vector $Q$ as $\pm \Delta_{\mathrm{PDW}}\left(\sin k_{x}+i \sin k_{y}\right)$ $c_{\boldsymbol{k}}^{\dagger} c_{-\boldsymbol{k}}^{\dagger}+$ H.c.. We set the parameters as $t_{0}=1, \mu=-1.25$, $\Delta_{\mathrm{PDW}}=0.052$, and $Q=\pi / 6$. In the left panel, we plot the spectral function in the original Brillouin zone. In the right panel, we plot the spectral function in the folded Brillouin zone (the region between the dashed lines in the left panel) with a new lattice constant $a_{Q}=a_{0} \times 2 \pi / Q$. Compared with Fig. 4 , the FS here can be viewed as a small perturbation of the original (circular) FS formed by composite fermions.

We set the parameters as $t_{0}=1, \mu=-1.25, \Delta_{\mathrm{PDW}}=0.82$, and $Q=\pi / 6$. The agreement between the computed spectral function $\rho(\boldsymbol{k}, E)$ and the FS analytically given by Eq. (3.26) is good, as shown in Fig. 4. The agreement becomes even better if we take $\mu \rightarrow-2$. In this case, the relevant dispersion becomes parabolic and approaches the continuum limit.

As $q \equiv m \Delta_{\text {PDW }}$ varies, the relative amplitudes of $t, \tilde{t}$, and $t^{\prime}$ vary periodically, and the twofold degenerate FS expands and shrinks. Note that at

$$
\sqrt{k_{F}^{2}-q^{2}} \equiv \kappa=n Q, \quad n \in \mathbb{Z}
$$

from Eq. (3.25), we have $t^{\prime}=0$ and $t=\tilde{t}$. Plugging these into Eq. (3.26), we see that the twofold degenerate FS shrinks to two Dirac points, both at $\boldsymbol{k}=0$. However, we see in the next subsection that the existence of two overlapping Dirac points, i.e., the fourfold degeneracy at $\boldsymbol{k}=0$, is a nonuniversal property of the continuum theory, and in generic cases at $\kappa=n Q$, the fermionic spectrum is actually gapped. To that end, we first need to understand whether and why the band crossing at the FS for generic values of $t$, $\tilde{t}$, and $t^{\prime}$ is robust.

Before we move on, let us briefly discuss the fermionic spectrum for $q \ll Q$. So far, we have worked in the regime where we only need to consider the nearest-neighbor coupling between the domain-wall Majorana modes. For $q \ll Q$, the domain-wall states are no longer well defined, as their localization length becomes longer than the PDW wavelength. In this case, the domain-wall Majorana modes are not a good starting point for analytical calculations. It turns out that this regime admits a simple description in $\boldsymbol{k}$ space. We note that due to Brillouin zone folding, the typical energy scale for the relevant bands in the folded $\mathrm{BZ}$ is given by $E_{F}^{Q} \sim v_{F} Q$. In this regime, we have $k_{F} \Delta_{\text {PDW }} \ll E_{F}^{Q}$, with $k_{F} \Delta_{\text {PDW }}$ being the size of the $p$-wave gap on the FS, which indicates that the PDW can be treated perturbatively in $\boldsymbol{k}$ space. Indeed, numerically, we find that the FS resembles that of the composite fermions, except at the regions with $k_{x}= \pm Q / 2, \pm 3 Q / 2, \ldots$, which becomes gapped and perturbatively reconstructed by the PDW order, as shown in Fig. 5.

Importantly, in this case, the FS are made out of Bogoliubov quasiparticles $d(\boldsymbol{k})=u_{\boldsymbol{k}} c(\boldsymbol{k})+v_{\boldsymbol{k}} c^{\dagger}(-\boldsymbol{k}+\boldsymbol{Q})$, which, in general, are not Majorana quasiparticles, i.e., $u_{k} \neq v_{k}$. For this reason, we call it the Bogoliubov FS to distinguish it from the Majorana FS that we obtained previously. As one increases $\Delta_{\mathrm{PDW}}$, the Bogoliubov FS gets progressively gapped and crosses over to the one obtained previously in Fig. 4.

\section{Symmetry-protected stability of the Majorana FS}

As we emphasized, two bands cross at the FS given by Eq. (3.26). It is then a natural question to ask whether this band crossing is robust against perturbations or is accidental due to the particular BdG Hamiltonian (3.1) we are using. Here, we show that the gapless nature of the FS is protected by symmetry. In particular, the defining inversion symmetry of the PDW state $\left|\Delta_{Q}\right|=\left|\Delta_{-Q}\right|$ again plays a crucial role.

In the literature, band crossings in $\boldsymbol{k}$ space that form submanifolds with co-dimension 2 and 3 have been intensively discussed. In two spatial dimensions, the band crossings are known as Dirac points, while in three spatial dimensions, they are Weyl points (with co-dimension 3), Dirac points (with co-dimension 3), and nodal lines (with co-dimension 2). The band crossing we obtain has codimension 1, which corresponds to nodal FSs. The stability of the nodal FS is less well known but has also been analyzed recently [66,75-79]. A particularly systematic analysis has been done in Ref. [77].

For our purposes, we closely follow the analysis in Ref. [66]. We focus on the particle-hole symmetry and the inversion symmetry previously identified for the BdG Hamiltonian (3.1) for the PDW state. With regard to the effective Hamiltonian Eq. (3.24), the particle-hole symmetry that relates positive and negative energy states of the Hamiltonian Eq. (3.24) is expressed through a unitary operator $\mathcal{C}$ :

$$
\begin{aligned}
\mathcal{C H}(-\boldsymbol{k})^{T} \mathcal{C}^{\dagger} & =-\mathcal{H}(\boldsymbol{k}), \\
\mathcal{C} & =\sigma_{0} \otimes \tau_{3}=\left(\begin{array}{cc}
\sigma_{0} & 0 \\
0 & -\sigma_{0}
\end{array}\right) .
\end{aligned}
$$


The effective Hamiltonian Eq. (3.24) also inherits the inversion symmetry of the PDW state under inversion operation $x \rightarrow-x$ with respect to the domain wall. Because the right- and left-moving states, Eq. (3.14), are formed by linear combinations of even- and odd-parity states, they are switched under inversion. Inversion symmetry is represented in the effective theory Eq. (3.24) by a unitary operator $\mathcal{I}$ satisfying

$$
\begin{aligned}
\mathcal{I}\left(-k_{x}\right) \mathcal{H}(-\boldsymbol{k}) \mathcal{I}\left(-k_{x}\right)^{\dagger} & =\mathcal{H}(\boldsymbol{k}), \\
\mathcal{I}\left(k_{x}\right) & =\left(\begin{array}{cc}
\sigma_{1} & 0 \\
0 & e^{-i k_{x}} \sigma_{1}
\end{array}\right),
\end{aligned}
$$

where the action of $\sigma_{1}$ is to switch left- and right-moving modes, and the momentum dependence $e^{-i k_{x}}$ on the ADW d.o.f. reflects the fact that the center of inversion is taken with respect to a DW. Both $\mathcal{C}$ and $\mathcal{I}$ relate $\boldsymbol{k}$ with $\boldsymbol{- k}$, and it is useful to consider their composite, which relates $\mathcal{H}(\boldsymbol{k})$ to itself. We define another unitary operator

$$
U_{\mathcal{C I}}\left(k_{x}\right) \equiv \mathcal{C} \mathcal{I}\left(k_{x}\right)=\left(\begin{array}{cc}
\sigma_{1} & 0 \\
0 & -e^{-i k_{x}} \sigma_{1}
\end{array}\right)
$$

which, importantly, is symmetric. It then follows that for any given $\boldsymbol{k}$,

$$
U_{\mathcal{C I}}\left(k_{x}\right) \mathcal{H}(\boldsymbol{k}) U_{\mathcal{C I}}^{\dagger}\left(k_{x}\right)=-\mathcal{H}^{T}(\boldsymbol{k}) .
$$

Any symmetric matrix can be diagonalized as $U_{\mathcal{C I}}=$ $Q \Lambda Q^{T}$, with $\Lambda$ diagonal and $Q$ unitary. Then, as shown in Ref. [66], Eq. (3.33) can be used to define an antisymmetric $\tilde{\mathcal{H}}(\boldsymbol{k})=\Omega(\boldsymbol{k}) \mathcal{H}(\boldsymbol{k}) \Omega^{\dagger}(\boldsymbol{k})$, with $\tilde{\mathcal{H}}(\boldsymbol{k})=-\tilde{\mathcal{H}}^{T}(\boldsymbol{k})$, where $\Omega(k)=\sqrt{\Lambda^{\dagger}} Q^{\dagger}$ is unitary. The antisymmetric nature of $\tilde{\mathcal{H}}(\boldsymbol{k})$ allows us to express the determinant at any given $\boldsymbol{k}$ in terms of the Pfaffian as

$$
\operatorname{det}[\mathcal{H}(\boldsymbol{k})]=\operatorname{det}[\tilde{\mathcal{H}}(\boldsymbol{k})]=\{\operatorname{Pf}[\tilde{\mathcal{H}}(\boldsymbol{k})]\}^{2} .
$$

At the location of the FS, $\operatorname{det}[\mathcal{H}(\boldsymbol{k})]=\operatorname{Pf}[\mathcal{H}(\boldsymbol{k})]=0$. Importantly, since $\mathcal{H}$ is Hermitian, one can check that the Pfaffian $\operatorname{Pf}[\tilde{\mathcal{H}}(\boldsymbol{k})]$ is always real. If two points at the BZ, $\boldsymbol{k}_{1}$ and $\boldsymbol{k}_{2}$, satisfy $\operatorname{Pf}\left[\tilde{\mathcal{H}}\left(\boldsymbol{k}_{1}\right)\right] \operatorname{Pf}\left[\tilde{\mathcal{H}}\left(\boldsymbol{k}_{2}\right)\right]<0$, then there is a FS separating $\boldsymbol{k}_{1}$ and $\boldsymbol{k}_{2}$ at which the Pfaffian changes sign. Symmetry-preserving perturbations can move the location of the FS in $\boldsymbol{k}$ space, but they cannot gap the spectrum unless the FS shrinks to zero.

Specifically, for our tight-binding Hamiltonian (3.24), one obtains

$\operatorname{Pf}[\tilde{\mathcal{H}}(\boldsymbol{k})]=\left(v_{y} k_{y}\right)^{2}+(t+\tilde{t})^{2}-4\left(t^{\prime 2}+t \tilde{t}\right) \cos ^{2}\left(k_{x} / 2\right)$.

The contour plots of the Pfaffian, Eq. (3.35), are plotted in Fig. 6. The condition $\operatorname{Pf}[\mathcal{H}(\boldsymbol{k})]=0$ indeed matches the location of the FS given by Eq. (3.26). The FS is stable in

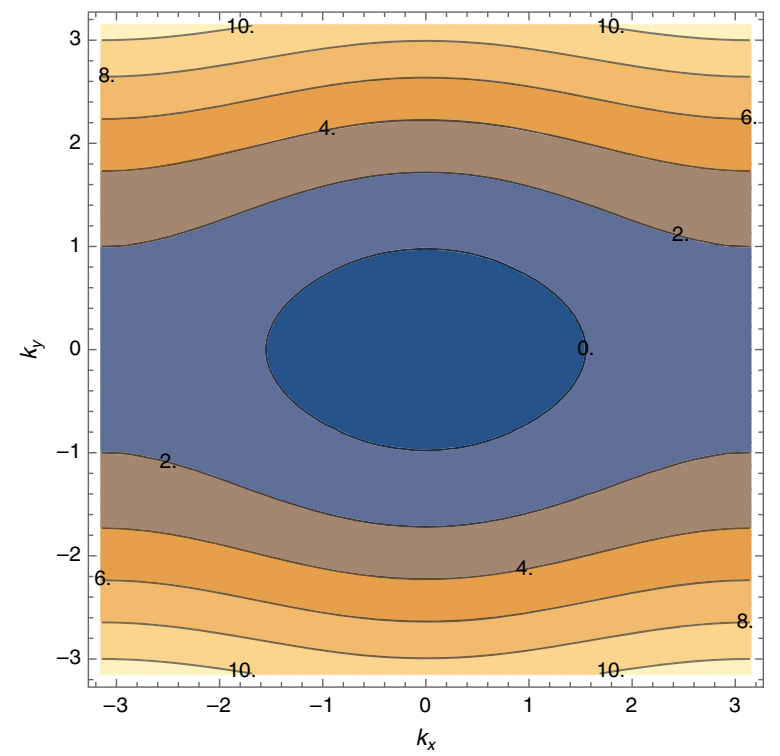

FIG. 6. Contour plots of the Pfaffian, Eq. (3.35), $t=0.4$, $t^{\prime}=0.5, \tilde{t}=0.4$, showing the FS for $\operatorname{Pf}[\tilde{\mathcal{H}}(\boldsymbol{k})]=0$, which separates the region where the Pfaffian changes sign. For parameter values such that $\kappa=n Q$, which correspond to $t^{\prime}=0$ and $t=\tilde{t}$, the FS shrinks to a doubly degenerate Dirac point at $\left(k_{x}, k_{y}\right)=(0,0)$. We note, however, that this Dirac point is accidental, in the sense that it is a property of the continuum approximation of the band structure in which the original FS (in the absence of a PDW order parameter) is circular. Our numerical calculation indeed shows that this Dirac point is gapped once lattice effects become nonnegligible.

the presence of small perturbations that preserve the two symmetries simultaneously.

\section{Gapped states from domain-wall coupling}

\section{Gapped phase near $\kappa=n Q$}

We continue to focus on the regime $Q<q<k_{F}$. Our argument on the grounds of inversion symmetry in Sec. III C establishes the stability of the doubly degenerate FS. However, it does not ensure the stability of the double Dirac points obtained from Eqs. (3.26) and (3.25) at $\kappa=n Q$, which in turn are obtained from the continuum BdG Hamiltonian, Eq. (3.1). Here, we show that for a BdG Hamiltonian with a generic lattice dispersion and $p$-wave form factor of local pairing, the Dirac spectra at $\kappa=n Q$ in the continuum model are replaced by gapped fermionic spectra. Moreover, remarkably, the gapped system has a trivial band topology, even though the local pairing symmetry is $p_{x}+i p_{y}$ with $\mu>0$.

It is instructive to first understand the origin of the double Dirac points at $\kappa=n Q$ in the continuum model. At these points, from Eq. (3.25), the same-chirality hopping amplitude $t^{\prime}$ vanishes. As a result, left movers only couple to their adjacent right movers and vice versa. The domainwall modes decompose into two separate chains of coupled 
wires, each of them alternating between left and right movers. We illustrate this situation in Fig. 8, where the solid arrowed lines denote $\tilde{t}$ and the dashed arrowed lines denote $t$. From Eq. (3.25), we see that, at $\kappa=n Q$, we have $t=\tilde{t}$. With $t=\tilde{t}$, each of the two chains gives rise to a Dirac point at $\boldsymbol{k}=0$, in a mechanism similar to the Dirac cone "reconstruction" at the surface of a topological insulator via hybridization of chiral modes localized at oppositely oriented ferromagnetic domain walls [80].

However, recall that, after a careful analysis, we have concluded that there is no symmetry that relates $t$ and $\tilde{t}$. The fact that we obtained $t=\tilde{t}$ in the continuum model at $\kappa=n Q$ is merely an accident. For a generic dispersion with an almost circular normal-state FS, we expect, from Eq. (3.25), that when $t^{\prime}=0$,

$$
\tilde{t}-t \propto N_{e}^{2}-N_{o}^{2}
$$

Following an analogy with the well-known Su-SchriefferHeeger model for polyacetylene [81], this asymmetrical coupling pattern gaps out the fermionic spectrum. The spectral gap is proportional to $N_{o}^{2}-N_{e}^{2}$. This spectral gap is rather small-in particular, for $q \ll k_{F}$, we have from Eq. (3.38) that $N_{e} \approx N_{o}$. For $q$ more comparable to $k_{F}$, this spectral gap increases. Naturally, in the vicinity of the would-be Dirac point values, i.e., near $\kappa=n Q$, the spectral gap persists, and for larger $q$ 's, the range of $q$ with a gapped spectrum is larger.

Indeed, we numerically solved the lattice version of the problem with Eqs. (3.27) and (3.28). With $t=1$ and $\mu=1.9$, the normal-state FS is nearly isotropic. Yet, we see that when the FS shrinks, it becomes gapped, instead of resulting in Dirac points. We show the gapped Dirac dispersion in this situation in Fig. 7(a). We have also verified that as $\Delta_{\text {PDW }}$ increases, the Dirac gap becomes larger.

The band topology of this gapped phase can be obtained by inspecting the edge modes. From Fig. 8, it is straightforward to see that the coupling pattern between the domain-wall modes (not including the leftmost and rightmost modes, which are edge modes) leaves two unpaired chiral domain-wall modes at the two ends. On the other hand, owing to the local $p_{x}+i p_{y}$ pairing symmetry, there would be a chiral mode (shown in yellow in Fig. 8) at each physical edge of the system. For $\tilde{t}>t$, i.e., when the hopping represented by solid arrowed lines is stronger, one can check that the unpaired domain-wall mode and the would-be edge mode are of opposite chirality, and they gap each other. The resulting state does not host any gapless edge modes and is thus topologically trivial with Chern number $\mathcal{C}=0$. On the other hand, if $t>\tilde{t}$, the unpaired domain-wall mode and the edge mode are of the same chirality; in this case, at each edge, there would be two chiral modes propagating in the same direction, with $\mathcal{C}=2$. A similar situation has been found in a $p$-wave $\mathrm{SC}$ in the presence of a vortex lattice [82].

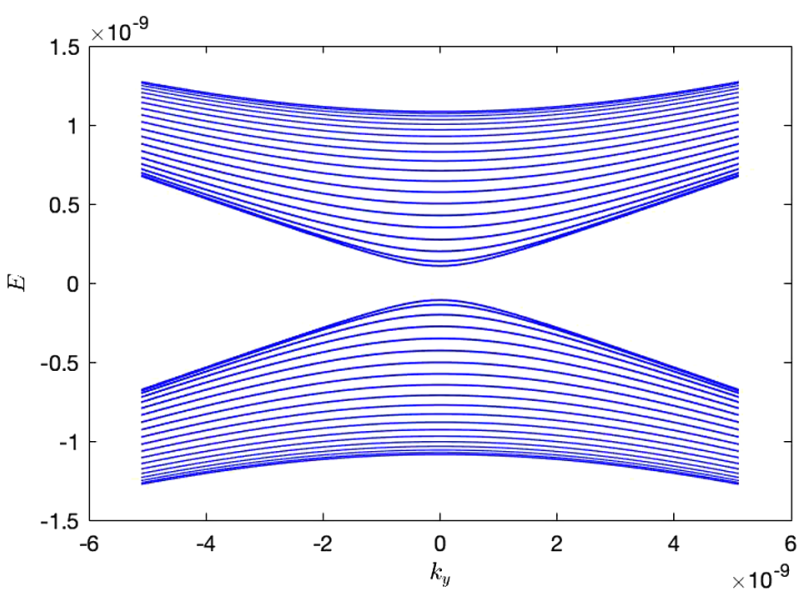

(a)

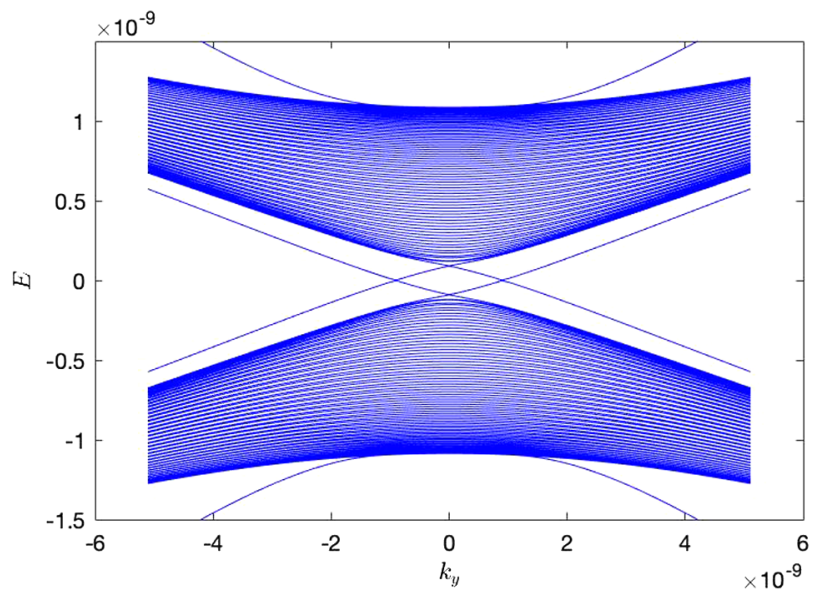

(b)

FIG. 7. The gapped Dirac spectrum from a numerical calculation near $\kappa=8 Q$. We use the lattice model in Eqs. (3.27) and (3.28), with $t=1$ and $\mu=1.9$. The chemical potential is very close to the band bottom; thus, the system is quasicontinuous, $k_{F} \ll 2 \pi / a$. Panel (a) is obtained by the periodic boundary condition in the $x$ direction, while panel (b) is from the open boundary condition. From counting the number of the localized edge modes in panel (b), such a phase has a Chern number $\mathcal{C}=2$. The smallness of the energy eigenvalues comes from the extreme tight-binding limit we take, $q \gg Q$, which ensures a quantitative match with analytical results.

It is also instructive to understand how the competition between $t$ and $\tilde{t}$ changes the Chern number by 2, by considering the following reasoning. Let $t^{\prime}=0, t=\tau+\delta$, and $\tilde{t}=\tau-\delta$; then, the effective Hamiltonian, after an appropriate unitary transformation, is in the form $\mathcal{H}=\boldsymbol{B}(\boldsymbol{k}) \cdot \boldsymbol{\Gamma}, \quad$ where $\quad \boldsymbol{B}(\boldsymbol{k})=\left[v_{y} k_{y},-2 \tau \sin \left(k_{x} / 2\right), 2 \delta\right.$ $\left.\cos \left(k_{x} / 2\right)\right]$ and $\boldsymbol{\Gamma}=\left(\Gamma_{1}, \Gamma_{2}, \Gamma_{3}\right)$ are anticommuting matrices with $\Gamma_{i}^{2}=1$. Then, at $\delta=0(t=\tilde{t})$, we see the two Dirac points at $\boldsymbol{k}=0$, which become massive for $\delta \neq 0$. The Chern number measures the winding of the spinor $\boldsymbol{B}(\boldsymbol{k})$ as $\boldsymbol{k}$ is varied. Importantly, the sign of $\delta$ controls the orientation of the spinor along the third axis (i.e., direction $\Gamma_{3}$ ). Reversing the sign of $\delta$ reverses the orientation of the 


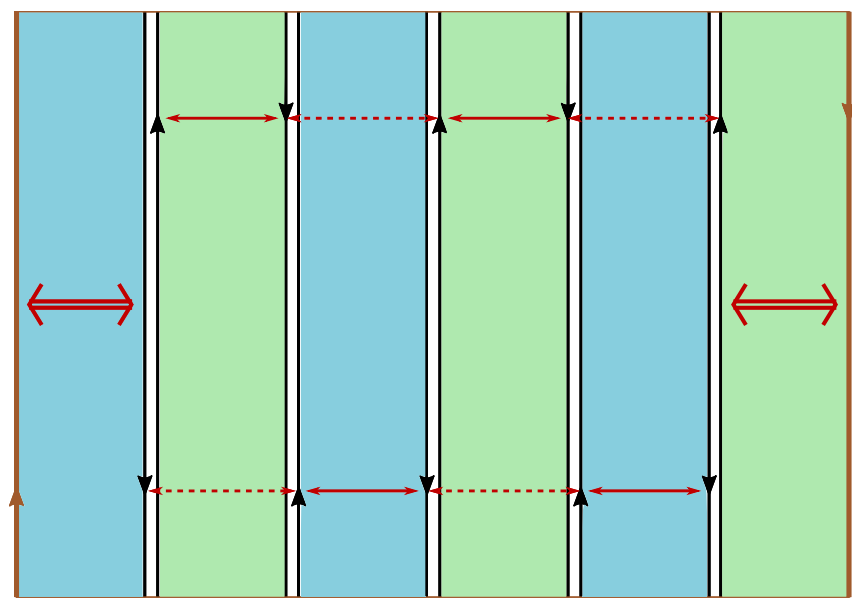

FIG. 8. Illustration of the gapped fermionic spectrum with Chern number $\mathcal{C}=0$ or $\mathcal{C}=2$, depending on whether $\tilde{t}$ (solid double lines) or $t$ (dashed double lines) is larger.

spinor and changes the Chern number by $\Delta C=2 \times 1=2$, where the factor of 2 accounts for the number of Dirac cones.

Notice that for both $\mathcal{C}=0$ and $\mathcal{C}=2$ pairing states, there are no Majorana zero modes bound at vortex cores. In particular, for the $\mathcal{C}=2$ state, there are two would-be zero modes near a vortex that generally gap each other. In our PDW setup, one of these would-be zero modes comes from the vortex core, and the other comes from a domain-wall mode circulating the vortex, as can be seen through an analysis similar to what we did for the edge modes. In terms of the quantum Hall physics, as we will see, the absence of the vortex Majorana modes indicates that these states have Abelian topological order.

For our square lattice model, from counting the number of edge modes in open boundary conditions, we find $\mathcal{C}=2$ at $\kappa=n Q$ points in the quasicontinuum limit. We show in Fig. 7(b) such a situation with $n=8$, with an open boundary condition in the $x$ direction. As can be seen, there are two propagating modes of each chirality. In Appendix $\mathrm{D}$, we compute the lattice corrections to $t, \tilde{t}$, and $t^{\prime}$ in Eq. (3.25) for our square lattice model, and we show that indeed $\tilde{t}>t$ at $t^{\prime}=0$. We have not done the calculation for other lattices, and from symmetry constraints alone, both $\mathcal{C}=0$ and $\mathcal{C}=2$ phases are possible. Quite remarkably, with a $p_{x}+i p_{y}$ local pairing symmetry, the PDW state realizes a band topology such as that for a $d+i d$ superconductor, even though their symmetry properties are very different.

\section{Gapped phase for $q>k_{F}$}

Now, we consider the hybridization of bound states with $q>k_{F}=\sqrt{2 m \mu}$ located at the nodes of the PDW order parameter. Here, we show that the bulk spectrum of the 2D array is gapped, and it is topologically trivial.
For $q>k_{F}$, as we mentioned, both Eqs. (3.10) and (3.25) continue to hold. The only difference is that now $\kappa$, $N_{o}$, and $\sin (\kappa x)$ are imaginary. It is convenient to express Eq. (3.25) in terms of real variables:

$$
\begin{aligned}
t= & -\frac{\bar{\kappa}}{4 m} \exp (-q \lambda / 2) \\
& \times\left[2 N_{e} \bar{N}_{o} \cosh \left(\frac{\bar{\kappa} \lambda}{2}\right)-\left(N_{e}^{2}+\bar{N}_{o}^{2}\right) \sinh \left(\frac{\bar{\kappa} \lambda}{2}\right)\right], \\
\tilde{t}= & -\frac{\bar{\kappa}}{4 m} \exp (-q \lambda / 2) \\
& \times\left[2 N_{e} \bar{N}_{o} \cosh \left(\frac{\bar{\kappa} \lambda}{2}\right)+\left(N_{e}^{2}+\bar{N}_{o}^{2}\right) \sinh \left(\frac{\bar{\kappa} \lambda}{2}\right)\right], \\
t^{\prime}= & \frac{\bar{\kappa}}{4 m} \exp (-q \lambda / 2)\left(N_{e}^{2}-\bar{N}_{o}^{2}\right) \sinh \left(\frac{\bar{\kappa} \lambda}{2}\right),
\end{aligned}
$$

where $\bar{\kappa} \equiv|\kappa|=\sqrt{q^{2}-k_{F}^{2}}$, and

$N_{e}=\sqrt{\frac{2 q\left(q^{2}-\bar{\kappa}^{2}\right)}{2 q^{2}-\bar{\kappa}^{2}}}, \quad \bar{N}_{o}=\sqrt{\frac{2 q\left(q^{2}-\bar{\kappa}^{2}\right)}{\bar{\kappa}^{2}}}$.

With these results, we notice that the Pfaffian of the spectrum (3.26),

$$
\begin{aligned}
& \operatorname{Pf}\left[\tilde{\mathcal{H}}\left(k_{x}, k_{y}\right)\right] \\
& =\left(v_{y} k_{y}\right)^{2}-4\left(t^{\prime 2}+t \tilde{t}\right) \cos ^{2}\left(k_{x} / 2\right)+(t+\tilde{t})^{2}>0,
\end{aligned}
$$

always holds for $q>k_{F}$. The proof is elementary: The Pfaffian is greater than $(t-\tilde{t})^{2}-4 t^{\prime 2} \propto \bar{N}_{o}^{2} N_{e}^{2}>0$. This result indicates that the fermionic spectrum is gapped for $q>k_{F}$. The size of the gap is of the same order as the $t$ 's. We remind the reader that the gap in the $q>k_{F}$ regime is typically larger than the gap in $q<k_{F}$ since the latter is given by lattice corrections (see Appendix D) and vanishes in the continuum limit.

The topology of this state can be obtained by a similar analysis at $q \gg k_{F}$. Since for smaller $q>k_{F}$ the gap does not close, the topology does not change. For $q \gg k_{F}, q \approx \bar{\kappa}$ and $N_{e} \approx \bar{N}_{o}$. Thus, from Eq. (3.37), we have $t^{\prime} \rightarrow 0$. Then, as we discussed previously in Sec. III D 1 and as shown in Fig. 8, the Chern number of this state again depends on the relative amplitude of $t$ and $\tilde{t}$. Here, from Eq. (3.37), we unambiguously have $t>\tilde{t}$, and therefore, the gapped state has $\mathcal{C}=0$; i.e., the band topology of the gapped state at $q>k_{F}$ is trivial.

It is worth comparing the trivial pairing state we obtained with the strong pairing phase considered in Ref. [59]. As we cautioned, the strong pairing there refers to a situation in which the normal state does not have a FS $(\mu<0)$. Both our state and the strong pairing phase are topologically trivial. In our case, however, we note that we always set $\mu>0$ in our state, so it may seem puzzling at first why our state is trivial. Here, the trivial topology is obtained by invoking additional domain-wall states, which by 
themselves couple into a 2D system that neutralizes the total Chern number.

\section{COEXISTENCE OF PDW ORDER AND UNIFORM PAIRING ORDER}

In this section, we focus on the fermionic spectrum in the presence of the coexisting PDW order parameter and the uniform $p_{x}+i p_{y}$ pairing order parameter. We refer to this state as the $p_{x}+i p_{y}$ stripe pairing state.

We determine the fermionic spectrum in the regime where the paired state has a $p_{x}+i p_{y}$ PDW state coexisting with a uniform component of the $p_{x}+i p_{y}$ pairing order. In general, we find that the fermionic spectrum is gapped. In particular, for $Q<q<k_{F}$, the Majorana FS is gapped as the inversion symmetry is broken. We analyze the band topology of the gapped phases and present a phase diagram.

\section{A. Gapping of the Majorana FS}

We assume that the order parameter of the uniform component has the same phase as the overall phase for the order parameter of the PDW state. The order parameter in real space is of the form

$\Delta(x)=\Delta_{\mathrm{u}}+\Delta_{\mathrm{PDW}}\left[1+\sum_{\epsilon=1,2} \sum_{n \in \mathbb{Z}}(-1)^{\epsilon} \operatorname{sgn}\left(x-x_{n}^{(\epsilon)}\right)\right]$,

where $\Delta_{\mathrm{u}}$ represents a uniform component of the order parameter. Crucially, we see that the inversion symmetry centered at the DWs and ADWs with $x=x_{n}^{(\epsilon)}$ are now broken by the uniform component $\Delta_{\mathrm{u}}$. A direct consequence is that the Majorana FS for $q<k_{F}$ protected by the particle-hole symmetry $\mathcal{C}$ and inversion symmetry $\mathcal{I}$ (Sec. III C) becomes gapped. Indeed, numerical calculations of Eq. (3.27) with both $\Delta_{\mathrm{u}}$ and $\Delta_{\mathrm{PDW}}$ confirm that the fermionic spectrum is gapped.

Instead of a detailed evaluation of the hopping matrices in a tight-binding Hamiltonian, like we did for Eq. (3.24), one can understand the gap opening in an intuitive way. In Appendix B, we show that the two zero-mode solutions obtained in Sec. III B persist so long as $\left|\Delta_{\mathrm{u}}\right|<\left|\Delta_{\text {PDW }}\right|$. With $\Delta_{\mathrm{u}}$, the domains and antidomains become imbalanced, with order parameters alternating between $\pm \Delta_{\text {PDW }}+\Delta_{\mathrm{u}}$, and we assume $\left|\Delta_{\mathrm{u}}\right|<\left|\Delta_{\text {PDW }}\right|$. As a direct result, the wave packets of the propagating-mode bound on a DW at $x=0$ also become asymmetric. Following a similar procedure leading to Eqs. (3.10) and (3.14),

$$
\begin{array}{r}
\left\langle\boldsymbol{r} \mid \Psi_{R}^{\prime}\right\rangle \propto \begin{cases}\exp \left[i k_{y} y-i \kappa_{+} x\right] \exp \left[-q_{+} x\right] & \text { for } x>0 \\
\exp \left[i k_{y} y-i \kappa_{-} x\right] \exp \left[q_{-} x\right] & \text { for } x<0,\end{cases} \\
\left\langle\boldsymbol{r} \mid \Psi_{L}^{\prime}\right\rangle \propto \begin{cases}\exp \left[i k_{y} y-i \kappa_{+} x\right] \exp \left[-q_{+} x\right] & \text { for } x>0 \\
\exp \left[i k_{y} y-i \kappa_{-} x\right] \exp \left[q_{-} x\right] & \text { for } x<0,\end{cases}
\end{array}
$$

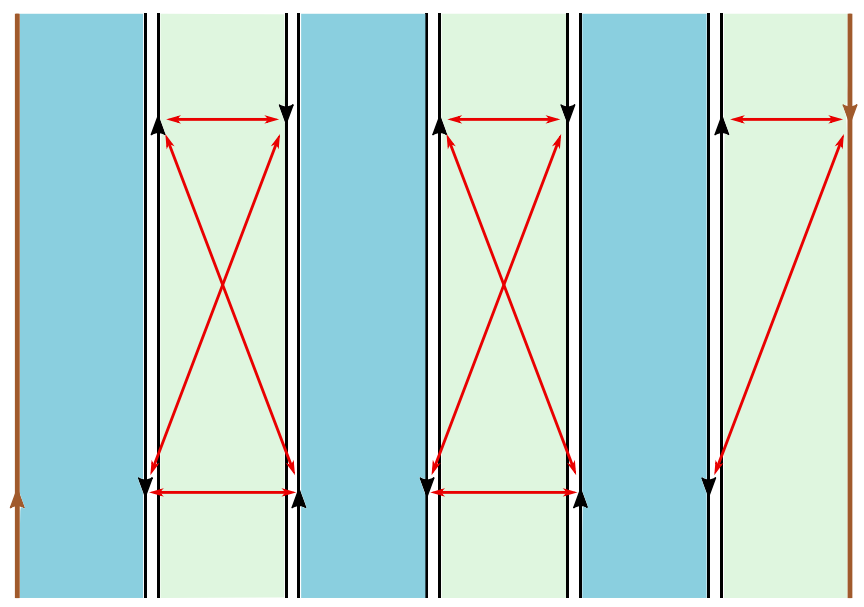

FIG. 9. Illustration of the gapped fermionic spectrum in the coexistence phase of PDW and uniform pairing with a Chern number $\mathcal{C}=1$.

where

$$
\begin{aligned}
& q_{ \pm} \equiv m\left(\Delta_{\mathrm{PDW}} \pm \Delta_{\mathrm{u}}\right), \\
& \kappa_{ \pm} \equiv \sqrt{k_{F}^{2}-q_{ \pm}^{2}} .
\end{aligned}
$$

Importantly, the wave packets of both left- and rightmoving modes are more extended into the domain where the order parameter has a smaller magnitude. Indeed, this result is expected since the local pairing order gaps out the local density of states and dictates the exponential decay of the wave packet.

Intuitively, the coupling between domain-wall states is stronger at regions with greater overlap of their wave functions. Analogous to the hopping amplitudes depicted in Fig. 2, one can define six hopping matrices, $t_{ \pm}, \tilde{t}_{ \pm}$, and $t_{ \pm}^{\prime}$, where \pm distinguishes domains with stronger or weaker local pairing order. Similar to Eq. (3.25), we have $t_{ \pm}, \tilde{t}_{ \pm}$, $t_{ \pm}^{\prime} \propto \exp \left(-q_{ \pm} \lambda / 2\right)$. In the tight-binding limit, we then have $t_{-} \ll t_{+}, t_{-}^{\prime} \ll t_{+}^{\prime}$, and $\tilde{t}_{-} \ll \tilde{t}_{+}$. In this limit, the system is "quadrumerized," with each quadrumer being composed of the left- and right-moving modes at a DWADW pair. We illustrate this case in Fig. 9. The quadrumerization develops in regions with a smaller pairing order and hence greater overlap between wave packets. Each quadrumer consists of two left movers and two right movers, and the hybridization in their wave functions leads to a gap.

It turns out that such a coexistence state has nontrivial band topology manifested by the presence of chiral edge states. In the quadrumer (tight-binding) limit, we consider a finite system (see Fig. 9). Depending on the termination of the finite system, near each physical edge, there is either one unpaired chiral mode (left edge in Fig. 9) or three would-be chiral modes (one edge mode and two nearby domain-wall modes, right edge in Fig. 9) with a net chirality. Either way, one gapless chiral mode survives at 
each physical edge. The existence of the stable gapless modes near the edges indicates that this coexistence state is topological and has a Chern number $\mathcal{C}=1$. It belongs to the same universality class as the weak-coupling regime in Ref. [59].

\section{B. Mean-field pairing phase diagram}

We end this section by placing all the phases mentioned above in a phase diagram in terms of the PDW order parameter $\Delta_{\mathrm{PDW}}$ and a possible coexisting uniform $p_{x}+i p_{y}$-wave order parameter $\Delta_{\mathrm{u}}$. We summarize the results in Fig. 10.

We have carefully analyzed the gapped phases in a pure PDW state, both for $q<k_{F}$ (or equivalently $\Delta_{\text {PDW }}<v_{F}$ ) and for $q>k_{F}$ (or equivalently $\Delta_{\mathrm{PDW}}>v_{F}$ ). Because of the spectral gap, these states are stable in the presence of a small $\Delta_{\mathrm{u}}$, which induces a "competing mass" that leads to a $\mathcal{C}=1$ phase. One naturally expects that the sizes of these phases in the $\Delta_{\mathrm{u}}$ direction are proportional to their spectral gaps. Therefore, in a semicontinuous limit where the lattice corrections are small, the $\mathcal{C}=2$ phase with $q<k_{F}$ occupies a much smaller region with $\Delta_{\mathrm{u}} \neq 0$ than the $\mathcal{C}=0$ phase with $q>k_{F}$ does. Both of these phase transitions involve a change in Chern number by 1 , and we have numerically verified that the phase transition occurs with a gap closing through a Dirac point at the phase boundaries.

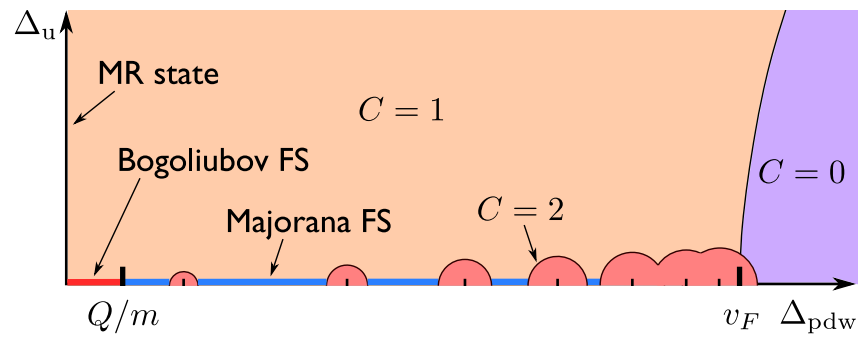

FIG. 10. Schematic pairing phase diagram for the fermionic states as a function of the PDW order parameter and a coexisting uniform $p$-wave order. When the uniform component $\Delta_{\mathrm{u}}=0$, the hybridization of the bulk domain walls, in general, gives rise to a FS for $\Delta_{\mathrm{PDW}}<v_{F}$. For $\Delta_{\mathrm{PDW}}<Q / m$ (or $\left.q<Q\right)$, the FS is from a perturbative reconstruction of the normal-state FS. The fermionic excitations are Bogoliubov quasiparticles. For $Q / m<\Delta_{\mathrm{PDW}}<v_{F}$, the FS is made of Majorana modes from the domain walls. We use the terms Bogoliubov FS and Majorana FS to distinguish them. Near specific values of $\Delta_{\text {PDW }}$ such that $\kappa=n Q$, (weak) lattice effects gap out the fermionic spectrum with a Chern number $\mathcal{C}=2$ (although $\mathcal{C}=0$ states may also be possible depending on lattice details). Above the critical pairing strength $\Delta_{\text {PDW }}>v_{F}$, the system enters a topologically trivial gapped state $(\mathcal{C}=0)$. This state survives a finite amount of uniform component $\Delta_{\mathrm{u}}$. The neutral FS becomes gapped for any $\Delta_{\mathrm{u}} \neq 0$, when the system enters the topological pairing phase $(\mathcal{C}=1)$ whose edge states contain a chiral Majorana mode. For $\Delta_{\mathrm{u}} \gg \Delta_{\text {PDW }}$, the system approaches a uniform $p$-wave state.

\section{THE $p_{x}+i p_{y}$ PDW FRACTIONAL QUANTUM HALL STATES}

Our study has so far addressed the properties of the fermion spectrum in a paired state and, as such, can be viewed as a description of a stripe superconductor with a chiral $p$-wave order parameter. We now turn on the implications of our results for the $\mathrm{FQH}$ physics of this state, keeping in mind that a paired $\mathrm{FQH}$ state is not a superconductor but in fact a charge insulator in an applied magnetic field. In order to make contact with the physics of the paired quantum Hall states, we reintroduce both charge and neutral modes on equal footing and recall that they are coupled to a dynamical Chern-Simons gauge field.

The neutral fermion modes we studied in the previous sections, which originated from a change in sign of the $p_{x}+i p_{y}$ order parameter, are akin to zero-energy Andreev bound states in a Josephson junction, where the difference in the phase of the order parameter is $\pi$. Just as an external magnetic flux alters the phase difference and gives rise to a spatially oscillating current passing through a Josephson junction [83], one might worry that the same would happen in this case due to the Chern-Simons and the external magnetic fields. The situation, however, is greatly simplified (at least in the mean-field description assumed here) due to the complete screening of the external magnetic flux by the Chern-Simons flux attached to the particles, which implies that the total effective magnetic field experienced by the composite fermions is zero and, thus, that the gauge fields do not alter the character of the Andreev bound states.

The discussion above can be made more concrete by recalling that at filling fraction $\nu=1 / 2$ of this $N=1 \mathrm{LL}$, upon performing a standard mapping to composite fermions coupled to a fluctuating Chern-Simons gauge field $a_{\mu}$, with $\mu=0,1,2$, the effective Lagrangian of the paired $\mathrm{FQH}$ state (in units where $e=\hbar=c=1$ ) reads

$$
\begin{aligned}
\mathcal{L}= & \psi^{\dagger}\left(i D_{0}+\mu\right) \psi+\frac{1}{2 m}|\boldsymbol{D} \psi|^{2}+\Delta \psi \psi \psi+\Delta^{*} \psi^{\dagger} \psi^{\dagger} \\
& +\frac{1}{4 \pi} \frac{1}{2} \epsilon^{\mu \nu \lambda} a_{\mu} \partial_{\nu} a_{\lambda},
\end{aligned}
$$

where $D_{\mu}=\partial_{\mu}+i\left(A_{\mu}+a_{\mu}\right)$ and, on average,

$$
\boldsymbol{\nabla} \times(\boldsymbol{a}+\boldsymbol{A})=0 .
$$

This condition defines the mean-field state and enforces that the electronic density $\rho=1 / 2$ everywhere in the bulk of the system. Had the total flux $\boldsymbol{\nabla} \times(\boldsymbol{a}+\boldsymbol{A})$ been nonzero in the region across the domain wall (which would have implied a local variation either of the magnetic field, the charge density, or both), then the associated Josephson effect would depend on the gauge-invariant phase difference across the junction (i.e., the domain wall) that carries a contribution from the gauge fields. However, in the meanfield state characterized by Eq. (5.2), the phase difference $\pi$ 


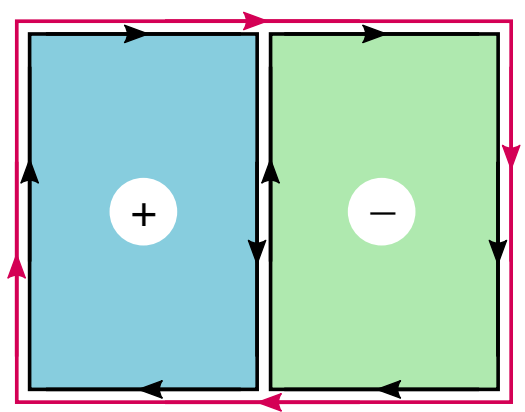

FIG. 11. Charge chiral mode at the boundary and Majorana modes, both at the edge and at the domain wall in the bulk of the system.

associated with the order parameter $\Delta$ fully specifies the properties of the low-energy states bound at the domain walls. To simplify the notation, in the Lagrangian of Eq. (5.1), the $p_{x}+i p_{y}$ symmetry structure of the pairing has been included in the pair field $\Delta$.

\section{A. Spectra of $p_{x}+i p_{y}$ PDW FQH states}

The bulk Chern-Simons term in Eq. (5.1) encodes the property that the system is a charge insulator in the bulk with a gapless chiral bosonic mode at the boundary of the system describing the charged excitations. The neutral fermion excitations of the system, either in the bulk or at the boundary, on the other hand, are described by the fermionic sector with the PDW order parameter. Thus, the neutral fermionic spectra of the stripe paired FQH states are those that we obtained for the $p_{x}+i p_{y}$ PDW state in Sec. III, while the charged bosonic sector is described by the Chern-Simons action. The stripe paired FQH system then has gapless neutral excitations supported at domain walls in the bulk of the system while remaining a bulk charge insulator with gapless charge modes on the edge, as illustrated in Fig. 11 (showing only two domains).

The analysis of Sec. III combined with the charge sector discussed above shows that there are four phases of the stripe paired FQH state, which are summarized in Fig. 1 and in the pairing phase diagram, Fig. 10. In the absence of a uniform $p_{x}+i p_{y}$-wave component $\Delta_{\mathrm{u}}$, when the Fermi energy is large compared to the pairing term of the PDW $\operatorname{order}\left(k_{F}>q\right.$, or equivalently $\left.v_{F}>\Delta_{\mathrm{PDW}}\right)$ and the system supports domain walls in the bulk, then the zero modes in each domain wall hybridize with their neighbors, giving rise to a 2D FS of charge-neutral Bogoliubov quasiparticles, a Majorana FS, as represented in Fig. 1(c). Quite remarkably, these neutral Majorana excitations are formed while the charged d.o.f. remain gapped (which implies that tunneling of electrons in the bulk is suppressed by the charge gap). This neutral FS implies that the system has an anisotropic unquantized bulk thermal conductivity and a heat capacity that scales linearly with temperature $T$, while its charge transport is gapped with a sharp plateau of $\sigma_{x y}=1 / 2$. This exotic "critical phase" is one of our central findings of this work.

A different paired stripe $\mathrm{FQH}$ state at $\nu=5 / 2$ was proposed by Wan and Yang [40], which is a state with alternating domains of Pfaffian and anti-Pfaffian states. Similar to our results, they found a state with gapped charge modes but gapless neutral modes at each domain wall. However, the domain wall between the Pfaffian and the anti-Pfaffian state has a more intricate structure than in the case of the $p_{x}+i p_{y}$ PDW state we propose here, leading to a more complex set of domain-wall modes. Moreover, the analysis of Ref. [40] neglects the coupling (and tunneling) between the neighboring domain-wall modes, which, as we showed here, plays an important role in the physics of the state. Thus, it is an open question whether these couplings will induce a bulk gap or not. In contrast, the gapless state obtained here survives the coupling between the domainwall modes, as it is protected by symmetry.

Furthermore, as indicated in the phase diagram, Fig. 10, for $k_{F}>q$, centered around each would-be Dirac point at $\sqrt{k_{F}^{2}-q^{2}}=n Q, n \in \mathbb{Z}$, there exists a gapped phase with Chern number $\mathcal{C}=2$ with two copropagating neutral models near the boundary (in addition to the charge mode). This $\mathcal{C}=2$ region represents an Abelian $\mathrm{FQH}$ state, as the vortices do not support Majorana zero modes. The edge CFT is composed of two chiral Majorana fermions and one charge mode, with a chiral central charge $c=2$. We are not aware of any previous discussions of this exotic $\mathrm{FQH}$ state.

The neutral FS is unstable towards gapped phases with distinct topological properties. The first type of instability happens in the weak pairing regime $\left(k_{F}>q\right)$, and it is triggered by a nonzero uniform component of the $p_{x}+i p_{y^{-}}$ wave order parameter $\Delta_{\mathrm{u}} \neq 0$. In this case, the neutral FS becomes topologically gapped with a Chern number $\mathcal{C}=1$, and the system is in the same universality class as the nonAbelian Pfaffian state. The transition between this state and the aforementioned $\mathcal{C}=2$ state is of Dirac type. Just like the Pfaffian state, in the bulk, there exists non-Abelian anyons with $e / 4$ electric charge, and the edge is described by a $U(1)_{2} \times$ Ising $/ \mathbb{Z}_{2}$ CFT with a chiral central charge $c=3 / 2$. The factor of $\mathbb{Z}_{2}$ accounts for gauge symmetry associated with representing the electron operator as a product of a Majorana fermion of the Ising sector and a charge-one vertex operator of the $U(1)_{2}$ sector.

Another instability of the neutral FS occurs at $\Delta_{\mathrm{u}}=0$ when the pairing potential is stronger than the Fermi energy, $q>k_{F}$. This transition is associated with a qualitative change in the character of the DW zero-mode states, as discussed in Sec. III B, which causes right- and leftmoving modes to display an asymmetric decay near the domains and, consequently, gaps both the bulk and the edge modes. This pairing phase is characterized by a Chern number $\mathcal{C}=0$. The disappearance of the neutral fermion modes from the low-energy spectrum indicates a transition from a non-Abelian state to an Abelian state [84], the latter 
in the universality class of the Halperin paired state [85], where electrons form tightly bound charge- 2 pairs that condense in an Abelian state with $\sigma_{x y}=1 / 2$.

Note that, unlike in Ref. [59], where the transition from the Pfaffian to the Abelian state only occurs at chemical potential $\mu=0$ for a spatially uniform order parameter, for the PDW state considered here, the critical phase occurs for a positive $\mu$ and for a finite range of the parameter $\Delta_{\text {PDW }}$. The strength of the PDW order parameter behaves as a new "knob" that tunes the system through that transition between different topological orders. As we explained, this striking stability of the neutral FS stems from the symmetries possessed by the PDW state, which restricts the coupling of the Majorana modes both within each domain wall and between domain walls.

\section{B. Phase structure near $\nu=5 / 2$}

We end with a qualitative discussion of the place of the $p_{x}+i p_{y}$-PDW FQH state in a global phase diagram of quantum Hall states. Much as in the case of other liquidcrystalline quantum Hall states [7], the $p_{x}+i p_{y}$-PDW $\mathrm{FQH}$ state can melt either quantum mechanically or thermally in a number of different ways, similar to the melting phase diagram conjectured for the PDW superconductor in Ref. [42], by a generalization of the wellknown theories of 2D classical melting [86-88].

In the case of the PDW superconductor (including a $p_{x}+i p_{y}$-PDW state), the different pathways are also determined by the proliferation of the panoply of its topological excitations. The $p_{x}+i p_{y}$ PDW, just as its $d$-wave cousin, has three types of topological excitations: quantized vortices, half-vortices bound to single dislocations, and double-dislocations [42]. The proliferation of quantized and/or half-vortices destroys the paired state and leads to two possible compressible unquantized states: either a charge stripe state or a compressible nematic phase.

On the other hand, the proliferation of double dislocations leads to a uniform incompressible state best described as a quartet $\mathrm{FQH}$ condensate. The quartet $\mathrm{FQH}$ condensate is an analog of the charge-4e superconductor [42], where four (rather than two) fermions form a bound state and condense. Strong arguments have been presented [89] that a quartet condensate (as well as a charge-4e topological superconductor) has Abelian topological order. A detailed analysis of the properties of the quartet $\mathrm{FQH}$ state, however, is beyond the scope of the present work.

However, the properties of the different resulting phases depend on features specific to the physics of the FQH states. In addition to the condensates (paired or not), FQH fluids have a dynamical emergent gauge field, the ChernSimons gauge field. One of the consequences of the emergent Chern-Simons gauge field being dynamical is that the vortices of the condensate (i.e., the fundamental quasiparticles of the FQH state) have finite energy, instead of the logarithmically divergent energy of a vortex of a neutral superfluid. On the other hand, the effective interaction between the vortices may be attractive (as in a type-I superconductor) or repulsive (as in a type-II superconductor). In addition, $\mathrm{FQH}$ vortices carry fractional charge, and hence, vortices also interact with each other through the Coulomb interaction. The interplay between these different interactions was analyzed in the context of uniform paired FQH states by Parameswaran and co-workers [90,91], who predicted a complex phase with different liquid-crystal phases depending on whether the FQH fluid is in a type-I or type-II regime.

Much of the analysis summarized above can be extended, with some caveats, to the case of the $p_{x}+i p_{y}$-PDW FQH state. One important difference vis-à-vis the PDW superconductors is that in a 2D system such as the 2DEG, in the absence of an underlying lattice, the dislocations of the associated charge order cost a finite amount of free energy. As such, they proliferate at any finite temperature, thus restoring translation invariance and resulting in a nematic phase at all nonzero temperatures [52]. This problem was considered before in the context of high-temperature superconductors in Ref. [53]. However, the presence of strong enough anisotropy, e.g., by uniaxial strain or by a tilted magnetic field, can trigger a phase transition to a state with unidirectional order, which can be a $p_{x}+i p_{y}$ PDW FQH state or a charge stripe state (the latter case was found in the DMRG numerical work of Zhu and co-workers [92]). Both of these stripe states thermally melt by proliferating dislocations, whose interactions are logarithmic in an anisotropic system [52].

The precise interplay between these (and other) phases depends on details of the length scales that govern quantum Hall fluids. It is widely believed (for good reasons) that, in the lowest Landau level, all length scales are approximately of the same order of magnitude as the magnetic length. In Landau levels $N \geq 1$ and higher, other scales may come into play. This fact is evinced by the recent experiments near $\nu=5 / 2$, which find an interplay between a (presumably uniform) paired state and a compressible nematic phase [28] and between a compressible nematic phase and a stripe phase (albeit in the $N=2$ Landau level) [16].

These additional length scales may affect the structure of the vortices and of the other topological excitations, and therefore the nature of the state obtained for fields and/or densities away from the precise value of the filling fraction $\nu=5 / 2$, but still inside the plateau for the incompressible state. More specifically, the $\mathrm{FQH}$ state has a fluctuating gauge field, with a Chern-Simons term and a (subdominant) Maxwell term, which introduces a screening length in the problem that will affect the structure of the vortices, type I or type II. This problem was considered before in the context of relativistic field theory [93] and, more relevant to our analysis, in the context of paired FQH states [90,91], although those works did not consider the interplay of a possible $p_{x}+i p_{y}$ paired state. For example, if a type-II 
regime becomes accessible, the vortex states may exhibit intertwined orders of analogous to those that arise in high$T_{c}$ superconductors $[94,95]$. In this case, a $p_{x}+i p_{y}$ PDW phase may arise in the vortex "halos" of the uniform paired state and could be stabilized close to $\nu=5 / 2$. The upshot of this analysis is that a complex phase diagram may yet be uncovered, beyond what has been seen in recent experiments.

\section{DISCUSSION AND CONCLUSION}

In this paper, we have studied the properties of a $2 \mathrm{D}$ pairdensity-wave state with a $p_{x}+i p_{y}$ chiral order parameter, which is periodically varying along one direction. We have shown that this physical system can support exotic bulk symmetry-protected (gapless or gapped) fermionic spectrum. This bulk gapless phase results from the hybridization of pairs of counterpropagating Majorana fermion states localized near the nodes of the order parameter. The stability of the Majorana states near the domain walls is a consequence of a combination of inversion and chiral symmetries associated with the unidirectional PDW order parameter.

In the weak-coupling regime (in the BCS sense) characterized by $v_{F}>\Delta_{\mathrm{PDW}}$, the zero modes are localized within the distance $q^{-1}$, where $q=m \Delta_{\mathrm{PDW}}$. We have shown that the hybridization of these domain-wall modes gives rise to a Majorana FS that is protected by both particle-hole and inversion symmetries and that the robustness of the FS can be captured by the properties of a Pfaffian. Our findings have been supported by an effective theory valid in the regime $q \gg Q$, in which the low-energy modes on adjacent domain walls hybridize weakly, as well as by numerical calculations in the regime where the domain walls strongly couple to many neighboring domain walls.

The FS obtained in the $v_{F}>\Delta_{\text {PDW }}$ regime is generically unstable to the presence of perturbations that break inversion symmetry. In particular, a small uniform of component the order parameter breaks the inversion symmetry that maps $\Delta_{\text {PDW }} \rightarrow-\Delta_{\text {PDW }}$ around a domain wall and destroys the FS, giving rise to a gapped spectrum of neutral fermionic excitations. Moreover, we have shown that this gapped phase is topological as it supports a chiral Majorana branch at the boundary of the system, which has the same topological properties of the uniform $p_{x}+i p_{y}$ paired state. Our analysis has also shown the existence of special points characterized by the condition $\sqrt{k_{F}^{2}-q^{2}}=n Q(n \in \mathbb{Z})$, for which FS becomes a Dirac point at $\left(k_{x}, k_{y}\right)=(0,0)$. This Dirac point is a consequence of the continuum approximation of the band structure and generically becomes gapped by distortions of the Majorana wave functions due to lattice effects, where the system, interestingly, has a fermionic spectrum with Chern number $\mathcal{C}=2$ and thus supports two edge Majorana modes. On the other hand, for the strong-coupling limit $\Delta_{\mathrm{PDW}}>v_{F}$, we found the resulting fermionic spectrum to be trivial. These findings have been summarized in the phase diagram, Fig. 10.

Viewed as a stripe superconductor, our theory shows the existence of zero-energy extended Majorana states in the bulk of a PDW phase with a chiral $p$-wave order parameter. In this case, all the excitations of the systems are neutral Majorana modes. We applied this theory to the paired $\mathrm{FQH}$ state at filling $\nu=5 / 2$ in which the composite fermions pair into a state with a spatially dependent order parameter. In fact, recent numerical work [31] has shown that, as a function of the 2DEG layer thickness, the effective interactions experienced by composite fermions in $N \geq 1$ Landau levels can give rise to a Pomeranchuk instability, which could account for a mechanism behind the formation of a nematic $\mathrm{FQH}$ state, as it is, in fact, in line with recent experimental findings [28].

In our description of the stripe $\mathrm{FQH}$ state at $\nu=5 / 2$, the charge modes remain gapped in the bulk and give rise to a chiral bosonic density mode at the boundary, which is a conformal field theory with central charge $c=1$. The PDW order parameter only changes the properties of the neutral fermionic sector.

From the discussion above, in the weak-coupling regime, the neutral particles develop and are gapless FS, protected by symmetry, while the bulk remains gapped to charge excitations. Consequently, while the tunneling of neutral (Majorana) quasiparticles is facilitated by the absence of an energy gap in the bulk, the tunneling of the electron is suppressed by the charge gap. Moreover, a nonzero uniform component gaps the neutral fermionic spectrum, and the system develops a chiral Majorana branch; we then identify this phase as a stripe Moore-Read state. At $\sqrt{k_{F}^{2}-q^{2}}=n Q$ points, the edge CFT includes two Majorana branches, and the topological order becomes Abelian. On the other hand, when the pairing effects become sufficiently strong, the system becomes gapped (even in the absence of a uniform component), and the system enters a phase without a neutral Majorana edge state; this phase is then identified with the stripe (Abelian) Halperin paired state.

We close with a discussion of the possible relation between the $p_{x}+i p_{y}$ PDW FQH state and the very recent experiments of Hossain and co-workers [41], whose results were recently published. This experiment considers a 2DEG in an AlAs heterostructure, which has two elliptical electron pockets oriented at $90^{\circ}$ from each other. Each pocket has very anisotropic effective masses, with a ratio of $5: 1$. Under a very weak unidirectional strain field, the Landau level of one or the other pocket is emptied, and the system has a strong electronic anisotropy. Importantly, in these systems, at the fields in which the experiments are done, the Zeeman energy is larger than the Landau gap, as is the energy splitting due to the applied strain.

Remarkably, the experiments of Ref. [41] find a clear plateau in the $N=1$ Landau level at $\nu=3 / 2$, equivalent to 
the much-studied $\nu=5 / 2$ plateau in the 2DEG in GaAsAlAs heterostructures. However, these authors also found a remarkable transport anisotropy inside the plateau regime, by which, below some well-defined temperature, the longitudinal resistance $R_{x x}$ [along the (100) direction] rises sharply to a value comparable to $R_{x y}$, while resistance $R_{y y}$ [along the $(0,1,0)$ direction] decreases sharply. This nematic behavior is reminiscent of the earlier findings of Xia and co-workers [32] near the filling fraction $\nu=7 / 3$ in the $N=1$ Landau level of the 2DEG in GaAs-AlAs heterostructures.

While it is tempting to interpret these experimental results as evidence for the existence of the $p_{x}+i p_{y}$ PDW FQH state, it also raises a question since the magnitude of the longitudinal resistance seems incompatible with this state, which has a bulk charge gap. We should note that this experiment cannot distinguish a nematic state (which is uniform) from any stripe state (which breaks translational symmetry), paired or not. There are several possible ways to understand this behavior. One is that for a sample with the form of a QH bar, the strain does not force the system into a single oriented domain; there may be two orthogonally oriented domains in the bar geometry. In this scenario, the longitudinal transport is only carried by the charge edge mode, and it is drastically anisotropic. Other scenarios are also possible, such as the one suggested by the analysis of Parameswaran and co-workers [91]; perhaps the paired state is in the type-I regime, which leads to a form of Coulomb-frustrated phase separation. However, in this latter scenario, it is hard to understand why $R_{x y}$ has a sharp plateau. At any rate, if the state found in these experiments is a $p_{x}+i p_{y}$ PDW FQH state, it should exhibit bulk thermal conduction, as predicted by our analysis.

In summary, we have presented a new scenario characterized by a $2 \mathrm{D}$ chiral topological phase being intertwined with a stripe order, in which low-energy neutral fermionic d.o.f. are found to be supported at the nodes of the PDW order parameter. Our findings have implications for the understanding of nematic paired $\mathrm{FQH}$ states at filling $\nu=5 / 2$, as well as for nematic (or stripe) superconductors.

\section{ACKNOWLEDGMENTS}

We thank Daniel Agterberg, Steven Kivelson, Ganpathy Murthy, Mansour Shayegan, and Ajit Srivastava for discussions. E. F. is particularly grateful to S. Kivelson for numerous discussions (and the suggestion for the interpretation of the anisotropic transport in the context of the $7 / 3$ state). This work was supported in part by the Gordon and Betty Moore Foundation EPiQS Initiative through Grant No. GBMF4305 at the University of Illinois (L. H.S. and Y.W.) and the National Science Foundation Grant No. DMR-1725401 at the University of Illinois (E. F.). L. H. S. and Y. W. performed part of this work at the Aspen Center for Physics, which is supported by National Science Foundation Grant No. PHY-1607611.

Note added.-Recently, we became aware of a preprint by Barkman and co-workers [96] who considered a timereversal-invariant $p$-wave superconductor consisting of alternating domains with $p_{x} \pm i p_{y}$ pairing. The physics of this state is very different from the time-reversal-breaking $p_{x}+i p_{y}$ PDW superconductor that we present in this paper.

\section{APPENDIX A: MAJORANA FERMIONS AT THE NODES OF THE PAIR-DENSITY-WAVE STATE}

Here, we provide details of the Majorana fermion states located at the nodes of the PDW order parameter. We begin with the BdG Hamiltonian of the paired state,

$$
H=\left(\begin{array}{cc}
\epsilon(\boldsymbol{p})-\mu & \frac{1}{2}\left\{p_{-}, \Delta\right\} \\
\frac{1}{2}\left\{p_{+}, \Delta^{*}\right\} & -\epsilon(\boldsymbol{p})+\mu
\end{array}\right),
$$

where $\epsilon(\boldsymbol{p})=\boldsymbol{p}^{2} / 2 m, \boldsymbol{p}=\left(p_{x}, p_{y}\right)=\left(-i \partial_{x},-i \partial_{y}\right)$ and $p_{ \pm}=p_{x} \pm i p_{y}=-i \partial_{ \pm}$(we set $\left.\hbar=1\right)$. The anticommutators appearing in the BdG Hamiltonian can be expressed as $\frac{1}{2}\left\{p_{-}, \Delta\right\}=-\frac{i}{2} \partial_{-} \Delta-i \Delta \partial_{-}$and $\frac{1}{2}\left\{p_{+}, \Delta^{*}\right\}=-\frac{i}{2} \partial_{+} \Delta^{*}-$ $i \Delta^{*} \partial_{+}$. The system is defined on the plane with $x \in$ $(-\infty, \infty)$ and $y \in(-L / 2, L / 2)$. The BdG Hamiltonian (A1) possesses a particle-hole symmetry (redundancy) $\sigma_{1} H \sigma_{1}=-H^{*}$, which relates positive and negative energy states: If $\Psi_{E}$ is an eigenmode of $H$ with energy $E$, then $\sigma_{1} \Psi_{E}^{*}$ is an eigenmode with energy $-E$. For an order parameter $\Delta(x) \in \mathbb{R}$, Hamiltonian (A1) is translation invariant along the $y$ direction, such that the momentum eigenmodes $\Psi_{E, k_{y}}(x, y)=e^{i k_{y} y} \phi_{E, k_{y}}(x)$ satisfy

$$
\begin{aligned}
& H_{k_{y}} \phi_{E, k_{y}}(x)=E \phi_{E, k_{y}}(x), \\
& H_{k_{y}}=\left(\begin{array}{cc}
\frac{-\partial_{x}^{2}+k_{y}^{2}}{2 m}-\mu & -\frac{i}{2} \Delta^{\prime}(x)+\Delta(x)\left(-i \partial_{x}-i k_{y}\right) \\
-\frac{i}{2} \Delta^{\prime}(x)+\Delta(x)\left(-i \partial_{x}+i k_{y}\right) & \frac{\partial_{x}^{2}-k_{y}^{2}}{2 m}+\mu
\end{array}\right) .
\end{aligned}
$$




\section{Zero modes}

We now consider an order parameter that changes sign at $x=0$, and we look for Majorana fermions supported along this domain wall. Particle-hole symmetry of Eq. (A1) implies that Majorana modes are described by

$$
\Psi_{0}^{\epsilon}=\left(\begin{array}{c}
\phi_{\epsilon} \\
(-1)^{\epsilon+1} \phi_{\epsilon}^{*}
\end{array}\right), \quad \epsilon=1,2
$$

(where $\epsilon$ denotes the zero-mode chirality), and, according to Eq. (A3), the zero-mode equation, after setting $k_{y}=0$ in Eq. (A2), simplifies to

$$
\left(-\frac{\partial_{x}^{2}}{2 m}-\mu\right) \phi_{1,2}(x) \mp i \Delta(x) \partial_{x} \phi_{1,2}^{*}(x) \mp \frac{i}{2} \Delta^{\prime}(x) \phi_{1,2}^{*}(x)=0 .
$$

We now consider the sharp domain-wall configuration

$$
\begin{aligned}
\Delta_{ \pm}(x) & =\mp \Delta_{\mathrm{PDW}} \operatorname{sgn}(x), \quad \Delta_{ \pm}^{\prime}(x)=\mp 2 \Delta_{\mathrm{PDW}} \delta(x), \\
\Delta_{\mathrm{PDW}} & >0
\end{aligned}
$$

and solve for the zero mode with positive or negative chiralities (the \pm label in the order parameter is intended to show that it is correlated with the zero-mode chirality). With the ansatz

$$
\Psi_{\epsilon}(x)=e^{i \pi / 4} \frac{\psi(x)}{\sqrt{2}}\left(\begin{array}{c}
1 \\
(-1)^{\epsilon+1} i
\end{array}\right) \equiv e^{i \pi / 4} \psi(x) u_{\epsilon},
$$

where $u_{\epsilon}$ are the eigenspinors of $\sigma_{y}$, the zero-mode equation (A4) reads

$$
\left(-\frac{\partial_{x}^{2}}{2 m}-\mu\right) \psi(x)-\Delta_{\mathrm{PDW}} \operatorname{sgn}(x) \partial_{x} \psi(x)-\Delta_{\mathrm{PDW}} \delta(x) \psi(x)=0 .
$$

We first consider $x<0$ and plug the ansatz $\psi_{L} \sim e^{i P_{L} x}$ into Eq. (A7), leading to $\left(P_{L}^{2} / 2 m\right)-\mu+i \Delta_{\mathrm{PDW}} P_{L}=0$, whose solutions are

$$
\begin{aligned}
P_{L} & =-i(q \pm \bar{\kappa}), \quad q=m \Delta_{\mathrm{PDW}}>0, \\
\bar{\kappa} & =\sqrt{q^{2}-2 m \mu}>0 .
\end{aligned}
$$

Then, as long as $\mu>0$, the solution is normalizable in the $x<0$ half-space, and the solution for $x<0$ reads $(\bar{\kappa} \equiv i \kappa)$

$\psi_{L}(x)=e^{q x}[A \cos (\kappa x)+B \sin (\kappa x)], \quad x<0$.

A similar approach to the $x>0$ region leads to

$$
\psi_{R}(x)=e^{-q x}[A \cos (\kappa x)+C \sin (\kappa x)], \quad x>0 .
$$

Notice that the continuity of $\psi(x)$ at $x=0$ fixes the same coefficient $A$ in Eqs. (A9) and (A10) and that the asymptotic form of $\psi_{L / R}$ for large values of $|x|$ guarantees that the solution is normalizable. We deal with the boundary condition at $x=0$ by integrating Eq. (A7) in an infinitesimal interval $(-\epsilon,+\epsilon)$ around $x=0$ and invoking continuity of $\psi(x)$ at $x=0$, which yields

$-\frac{1}{2 m} \lim _{\epsilon \rightarrow 0}\left[\psi^{\prime}(+\epsilon)-\psi^{\prime}(-\epsilon)\right]-\Delta_{\mathrm{PDW}} \psi(0)=0$.

\section{Majorana fermions for $q<\boldsymbol{k}_{\boldsymbol{F}}$}

For $k_{F}^{2} \equiv 2 m \mu>q^{2}$, there are two orthonormal zeroenergy solutions for a given $\epsilon=1,2$ :

$\Psi_{\epsilon, e}(x)=N_{e} \frac{e^{-q|x|}}{\sqrt{L}} \cos (\kappa x) u_{\epsilon}, \quad N_{e}=\sqrt{\frac{2 q\left(\kappa^{2}+q^{2}\right)}{\kappa^{2}+2 q^{2}}}$,

and

$\Psi_{\epsilon, o}(x)=N_{o} \frac{e^{-q|x|}}{\sqrt{L}} \sin (\kappa x) u_{\epsilon}, \quad N_{o}=\sqrt{\frac{2 q\left(\kappa^{2}+q^{2}\right)}{\kappa^{2}}}$.

\section{Majorana fermions for $q>\boldsymbol{k}_{\boldsymbol{F}}$}

For $q>k_{F}$, the expressions in Eqs. (A12a) and (A12b) are still correct, but it is convenient to reexpress them in real parameters:

$\Psi_{\epsilon, e}(x)=\bar{N}_{e} \frac{e^{-q|x|}}{\sqrt{L}} \cosh (\bar{\kappa} x) u_{\epsilon}, \quad \bar{N}_{e}=\sqrt{\frac{2 q\left(q^{2}-\bar{\kappa}^{2}\right)}{2 q^{2}-\bar{\kappa}^{2}}}$,

and

$\Psi_{\epsilon, o}(x)=\bar{N}_{o} \frac{e^{-q|x|}}{\sqrt{L}} \sinh (\bar{\kappa} x) u_{\epsilon}, \quad \bar{N}_{o}=\sqrt{\frac{2 q\left(q^{2}-\bar{\kappa}^{2}\right)}{\bar{\kappa}^{2}}}$.

(A13b)

\section{APPENDIX B: STABILITY OF THE ZERO MODES IN THE PRESENCE OF A SMALL UNIFORM $p$-WAVE COMPONENT}

In the presence of a uniform component $\Delta_{\mathrm{u}}$ of the order parameter, the domain wall is described by 


$$
\Delta(x)=\Delta_{\mathrm{u}}-\Delta_{\mathrm{PDW}} \operatorname{sgn}(x),
$$

with $\Delta_{\mathrm{u}}>0$ and $\Delta_{\mathrm{PDW}}>0$. We now show that the zeroenergy solutions are stable as long as $\Delta_{\mathrm{u}}<\Delta_{\text {PDW }}$. To see this stability, we note that for $x<0$, the order parameter is $\Delta_{\mathrm{PDW}}+\Delta_{\mathrm{u}} \equiv \Delta_{L}$, and for $x>0$, we have $-\Delta_{\mathrm{PDW}}+$ $\Delta_{\mathrm{U}} \equiv-\Delta_{R}$, where $\Delta_{L / R}>0$. Defining $q_{L}=m \Delta_{L}$, $q_{R}=m \Delta_{R}, \quad \kappa_{L}=\sqrt{2 m \mu-q_{L}^{2}}, \quad$ and $\kappa_{R}=\sqrt{2 m \mu-q_{R}^{2}}$, the zero-mode solutions have the form

$x<0: \psi(x)=e^{q_{L} x}\left[A \cos \left(\kappa_{L} x\right)+B \sin \left(\kappa_{L} x\right)\right] / \sqrt{L}$,

$x>0: \psi(x)=e^{-q_{R} x}\left[A \cos \left(\kappa_{R} x\right)+C \sin \left(\kappa_{R} x\right)\right] / \sqrt{L}$,

and they satisfy the condition

$-\frac{1}{2 m} \lim _{\epsilon \rightarrow 0}\left[\psi^{\prime}(+\epsilon)-\psi^{\prime}(-\epsilon)\right]-\Delta_{\mathrm{PDW}} \psi(0)=0$,

which implies

$$
\kappa_{R} C=\kappa_{L} B .
$$

We then identify two orthogonal solutions $\psi_{1}(x)$ and $\psi_{2}(x)$ given by

$\psi_{1}(x)=A\left[\Theta(-x) e^{q_{L} x} \cos \left(\kappa_{L} x\right)+\Theta(x) e^{-q_{R} x} \cos \left(\kappa_{R} x\right)\right]$,

$\psi_{2}(x)=B\left[\Theta(-x) e^{q_{L} x} \sin \left(\kappa_{L} x\right)+\frac{\kappa_{L}}{\kappa_{R}} \Theta(x) e^{-q_{R} x} \sin \left(\kappa_{R} x\right)\right]$,

where $A$ and $B$ are normalization constants that can be readily determined.

Notice that, in the limit $\Delta_{\mathrm{u}} \rightarrow 0$, the solutions above reduce to the even- and odd-parity solutions obtained before.

\section{APPENDIX C: DERIVATION OF THE TIGHT- BINDING EFFECTIVE HAMILTONIAN (3.24)}

Let us consider a tight-binding variational state

$$
\left|\Psi_{k_{x}, k_{y}}\right\rangle=\sum_{\epsilon=1,2} \sum_{\mu=L, R} c_{\epsilon, \mu} \sum_{n \in \mathbb{Z}} \frac{e^{i k_{x} n \lambda}}{\sqrt{N}}\left|\Psi_{\epsilon, \mu, n}\left(k_{y}\right)\right\rangle,
$$

where the subscript $\epsilon=1$ denotes DW modes and $\epsilon=2$ ADW modes. Recall that $\left|\Psi_{\epsilon=1}\right\rangle \propto\left|u_{1}\right\rangle=(1, i)^{T} / \sqrt{2}$ and $\left|\Psi_{\epsilon=2}\right\rangle \propto\left|u_{2}\right\rangle=(1,-i)^{T} / \sqrt{2}$. The coefficients $c_{\epsilon, \mu}$ are variational parameters, which are determined by minimizing the energy $E_{\boldsymbol{k}}=\left\langle\Psi_{k_{x}, k_{y}}|H| \Psi_{k_{x}, k_{y}}\right\rangle /\left\langle\Psi_{k_{x}, k_{y}} \mid \Psi_{k_{x}, k_{y}}\right\rangle$, which yields the secular equation

$$
\operatorname{det}\left[\mathcal{O}^{-1}(\boldsymbol{k}) \mathcal{H}(\boldsymbol{k})-E_{\boldsymbol{k}} I_{4 \times 4}\right]=0,
$$

where

$$
\begin{gathered}
\mathcal{O}(\boldsymbol{k})_{\varepsilon_{1} \mu_{1}, \varepsilon_{2} \mu_{2}}=\sum_{n} e^{i k_{x} n}\left\langle\Psi_{\varepsilon_{1} \mu_{1},-n}\left(k_{y}\right) \mid \Psi_{\varepsilon_{2} \mu_{2}, 0}\left(k_{y}\right)\right\rangle \\
\mathcal{H}(\boldsymbol{k})_{\varepsilon_{1} \mu_{1}, \varepsilon_{2} \mu_{2}}=\sum_{n} e^{i k_{x} n}\left\langle\Psi_{\varepsilon_{1} \mu_{1},-n}\left(k_{y}\right)\right| E_{\mu_{2}}\left(k_{y}\right)+V_{\text {total }}(x) \\
-v_{0}^{\left(\varepsilon_{2}\right)}(x)\left|\Psi_{\varepsilon_{2} \mu_{2}, 0}\left(k_{y}\right)\right\rangle \\
V_{\text {total }}(x)=\frac{1}{2}\left\{-i \partial_{x}, \Delta_{\text {total }}(x)\right\} \sigma_{x} \\
v_{0}^{\left(\varepsilon_{2}\right)}(x)=\frac{1}{2}\left\{-i \partial_{x}, \Delta_{0}^{\left(\varepsilon_{2}\right)}(x)\right\} \sigma_{x},
\end{gathered}
$$

$$
\begin{aligned}
\Delta_{\text {total }}(x)= & \Delta_{\mathrm{PDW}}+\sum_{n} \Delta_{n}^{(1)}(x)+\sum_{n} \Delta_{n}^{(2)}(x) \\
= & \Delta_{\mathrm{PDW}}-\sum_{n} \Delta_{\mathrm{PDW}} \operatorname{sgn}(x-n \lambda) \\
& +\sum_{n} \Delta_{\mathrm{PDW}} \operatorname{sgn}(x-(n+1 / 2) \lambda) .
\end{aligned}
$$

We first notice that, because DWs and ADWs are, respectively, proportional to the orthonormal spinors $\left|u_{1}\right\rangle$ and $\left|u_{2}\right\rangle$, the overlap matrix $\mathcal{O}$ is the identity matrix to leading order:

$$
\mathcal{O}=I_{4 \times 4}+\mathrm{O}\left(e^{-q \lambda}\right) .
$$

Then, according to Eq. (C2), the band structure is obtained by diagonalizing the Hermitian matrix

$$
\mathcal{H}\left(k_{x}, k_{y}\right)=\left(\begin{array}{llll}
\mathcal{H}_{1 R, 1 R} & \mathcal{H}_{1 R, 1 L} & \mathcal{H}_{1 R, 2 R} & \mathcal{H}_{1 R, 2 L} \\
\mathcal{H}_{1 L, 1 R} & \mathcal{H}_{1 L, 1 L} & \mathcal{H}_{1 L, 2 R} & \mathcal{H}_{1 L, 2 L} \\
\mathcal{H}_{2 R, 1 R} & \mathcal{H}_{2 R, 1 L} & \mathcal{H}_{2 R, 2 R} & \mathcal{H}_{2 R, 2 L} \\
\mathcal{H}_{2 L, 1 R} & \mathcal{H}_{2 L, 1 L} & \mathcal{H}_{2 L, 2 R} & \mathcal{H}_{2 L, 2 L}
\end{array}\right) .
$$

\section{Diagonal matrix elements of Eq. (C7)}

For $\mathcal{H}_{1 R, 1 R}$, we have

$$
\begin{aligned}
\mathcal{H}_{1 R, 1 R}\left(k_{x}, k_{y}\right)= & \sum_{n} e^{i k_{x} \lambda n} \int_{x} \Psi_{1 R}^{*}(x+n \lambda) \times\left\langle u_{1}\right|\left[E_{R}\left(k_{y}\right)\right. \\
& \left.+V_{\text {total }}(x)-v^{(1)}(x)\right]\left|u_{1}\right\rangle \Psi_{1 R}(x) \\
= & E_{R}\left(k_{y}\right) \sum_{n} e^{i k_{x} \lambda n} \int_{x} \Psi_{1 R}^{*}(x+n \lambda) \phi_{1 R}(x) \\
= & E_{R}\left(k_{y}\right)\left[1+\mathrm{O}\left(e^{-q \lambda}\right)\right] \approx E_{R}\left(k_{y}\right), \quad(\mathrm{C} 8
\end{aligned}
$$

where, in passing from the first to the second line, we used $\left\langle u_{1}\left|\left[V_{\text {total }}(x)-v^{(1)}(x)\right]\right| u_{1}\right\rangle=0$ since $V_{\text {total }}(x)-v^{(1)}(x)$ is proportional to the Pauli matrix $\sigma_{x}$. A similar calculation leads to the expression for the diagonal matrix elements of Eq. (C7):

$\mathcal{H}_{1 R / L, 1 R / L}= \pm E_{R}\left(k_{y}\right), \quad \mathcal{H}_{2 R / L, 2 R / L}= \pm E_{R}\left(k_{y}\right)$. 


\section{Off-diagonal matrix elements of Eq. (C7)}

By the same reasoning as before, we can show that, to leading order, the following matrix elements are zero:

$$
\mathcal{H}_{1 R / L, 1 L / R}=0, \quad \mathcal{H}_{2 R / L, 2 L / R}=0 .
$$

We are then left with the nonzero off-diagonal matrix elements of Eq. (C7), $\mathcal{H}_{2 \mu_{2}, 1 \mu_{1}}(k)$, with $\mu_{1}, \mu_{2}=R / L$. To leading order,

$$
\mathcal{H}_{2 \mu_{2}, 1 \mu_{1}}\left(k_{x}, k_{y}\right)=R_{\mu_{2}, \mu_{1}} e^{i k_{x} \lambda}+S_{\mu_{2}, \mu_{1}}+\mathrm{O}\left(e^{-q \lambda}\right),
$$

where

$$
\begin{aligned}
R_{\mu_{2}, \mu_{1}}= & -\Delta_{\mathrm{PDW}} \int_{-\infty}^{-\lambda / 2} d x\left[\Psi_{2 \mu_{2}}^{*}(x+\lambda / 2) \partial_{x} \Psi_{1 \mu_{1}}(x)\right. \\
& \left.-\partial_{x} \Psi_{2 \mu_{2}}^{*}(x+\lambda / 2) \Psi_{1 \mu_{1}}(x)\right] \\
S_{\mu_{2}, \mu_{1}}= & \Delta_{\mathrm{PDW}} \int_{\lambda / 2}^{\infty} d x\left[\Psi_{2 \mu_{2}}^{*}(x-\lambda / 2) \partial_{x} \Psi_{1 \mu_{1}}(x)\right. \\
& \left.-\partial_{x} \Psi_{2 \mu_{2}}^{*}(x-\lambda / 2) \Psi_{1 \mu_{1}}(x)\right] .
\end{aligned}
$$

Evaluation of the integrals in Eq. (C12) gives the effective Hamiltonian

$$
\begin{aligned}
& \mathcal{H}\left(k_{x}, k_{y}\right) \\
& =\left(\begin{array}{cccc}
v_{y} k_{y} & 0 & t^{\prime}+t^{\prime} e^{-i k_{x}} & t-\tilde{t} e^{-i k_{x}} \\
0 & -v_{y} k_{y} & -\tilde{t}+t e^{-i k_{x}} & t^{\prime}+t^{\prime} e^{-i k_{x}} \\
t^{\prime}+t^{\prime} e^{i k_{x}} & -\tilde{t}+t e^{i k_{x}} & v_{y} k_{y} & 0 \\
t-\tilde{t} e^{i k_{x}} & t^{\prime}+t^{\prime} e^{i k_{x}} & 0 & -v_{y} k_{y}
\end{array}\right),
\end{aligned}
$$

where the parameters $t, \tilde{t}$, and $t^{\prime}$ are given by Eq. (3.25).

\section{APPENDIX D: LATTICE CORRECTIONS TO THE HOPPING MATRICES $t, \tilde{t}$, AND $t^{\prime}$}

In this Appendix, we compute the leading-order corrections to Eq. (3.25) by an underlying square lattice. We focus on the quasicontinuous limit, where the Fermi wavelength $\lambda_{F} \equiv 2 \pi / k_{F}$ is much larger than the lattice constant $a$.

The wave function of the domain-wall modes can be obtained by solving the lattice version of Eq. (3.4), and by using a exponential function ansatz, the even- and oddparity wave functions still satisfy Eq. (3.10); only the expressions for $q, \kappa$, and $N_{o, e}$ are different from their continuum version. By a simple analysis, these lattice corrections are of $O\left[\left(k_{F} a\right)^{2}\right]$.

We recall that the hopping amplitudes were obtained by an integral. For example, for $t^{\prime}$,

$$
\begin{aligned}
t^{\prime}= & -\Delta_{\mathrm{PDW}} \int_{\lambda / 2}^{\infty} d x\left(\left[\partial_{x} \Psi_{L}^{*}(\boldsymbol{r})\right] \Psi_{R}(\boldsymbol{r}-\lambda / 2)-\Psi_{L}^{*}(\boldsymbol{r})\right. \\
& \left.\times\left[\partial_{x} \Psi_{R}(\boldsymbol{r}-\lambda / 2)\right]\right) .
\end{aligned}
$$

For a lattice system, first one needs to replace $\partial_{x}$ with its lattice version $i \sin \left(\hat{k}_{x}\right)$; doing so introduces corrections of $O\left[\left(k_{F} a\right)^{2}\right]$. In addition, one should replace the integral with summations at the lattice sites. The leading correction from this replacement can be obtained from the Euler-Maclaurin formula

$$
\begin{aligned}
\int_{\lambda / 2}^{\infty} f(x) d x= & a\left[f\left(\frac{\lambda}{2}+\frac{a}{2}\right)+f\left(\frac{\lambda}{2}+\frac{3 a}{2}\right)+\cdots\right] \\
& +a^{2} f^{\prime}\left(\frac{\lambda}{2}+\frac{a}{2}\right)+O\left(a^{3}\right) .
\end{aligned}
$$

Then, including the leading-order Euler-Maclaurin correction, $t^{\prime}$ is found to be

$$
\begin{aligned}
t^{\prime}= & -\frac{\kappa}{4 m} \exp \left(-\frac{q \lambda}{2}\right)\left(N_{e}^{2}+N_{o}^{2}\right) \sin \left(\frac{\kappa \lambda}{2}\right) \\
& -\frac{q^{2}}{2 m} \exp \left(-\frac{q \lambda}{2}\right)\left[N_{e}^{2}(2 \delta(0)+q)+2 N_{e} N_{o} \kappa\right] a^{2} .
\end{aligned}
$$

Regularizing $\delta(0)=1 / a$, we see that the leading correction to $t^{\prime}$ is of $O\left(k_{F} a\right)\left(q \lesssim k_{F}\right)$, given by the $\delta$-function term. We do not need to keep all other $O\left[\left(k_{F} a\right)^{2}\right]$ terms. Including the lattice corrections for all couplings, we have

$$
\begin{aligned}
t= & -\frac{\kappa}{4 m} \exp \left(-\frac{q \lambda}{2}\right) \\
& \times\left[2 N_{e} N_{o} \cos \left(\frac{\kappa \lambda}{2}\right)+\left(N_{e}^{2}-N_{o}^{2}\right) \sin \left(\frac{\kappa \lambda}{2}\right)\right] \\
& -\frac{q^{2} a}{m} \exp \left(-\frac{q \lambda}{2}\right) N_{e}^{2}, \\
\tilde{t}= & -\frac{\kappa}{4 m} \exp \left(-\frac{q \lambda}{2}\right) \\
& \times\left[2 N_{e} N_{o} \cos \left(\frac{\kappa \lambda}{2}\right)-\left(N_{e}^{2}-N_{o}^{2}\right) \sin \left(\frac{\kappa \lambda}{2}\right)\right] \\
& -\frac{q^{2} a}{m} \exp \left(-\frac{q \lambda}{2}\right) N_{e}^{2}, \\
t^{\prime}= & -\frac{\kappa}{4 m} \exp \left(-\frac{q \lambda}{2}\right)\left(N_{e}^{2}+N_{o}^{2}\right) \sin \left(\frac{\kappa \lambda}{2}\right) \\
& -\frac{q^{2} a}{m} \exp \left(-\frac{q \lambda}{2}\right) N_{e}^{2} .
\end{aligned}
$$

In the main text, we are interested in the case where $t^{\prime}=0$. It is straightforward to verify that, in this case, $\sin (\kappa \lambda / 2)<0$, and $\tilde{t}>t$. From the criterion given in the main text, the Chern number of this phase is $\mathcal{C}=2$. 
[1] R. E. Prange and S. M. Girvin, The Quantum Hall Effect (Springer-Verlag, Heidelberg, 1987).

[2] R. Willett, J. P. Eisenstein, H. L. Störmer, D. C. Tsui, A. C. Gossard, and J.H. English, Observation of an EvenDenominator Quantum Number in the Fractional Quantum Hall Effect, Phys. Rev. Lett. 59, 1776 (1987).

[3] W. Pan, J. S. Xia, V. Shvarts, D. E. Adams, H. L. Störmer, D. C. Tsui, L. N. Pfeiffer, K. W. Baldwin, and K. W. West, Exact Quantization of the Even-Denominator Fractional Quantum Hall State at $\nu=5 / 2$ Landau Level Filling Factor, Phys. Rev. Lett. 83, 3530 (1999).

[4] G. Moore and N. Read, NonAbelions in the Fractional Quantum Hall Effect, Nucl. Phys. B360, 362 (1991).

[5] C. Nayak and F. Wilczek, $2 n$ Quasihole States Realize $2^{n-1}$ Dimensional Spinor Braiding Statistics in Paired Quantum Hall States, Nucl. Phys. B479, 529 (1996).

[6] S. A. Kivelson, E. Fradkin, and V. J. Emery, Electronic Liquid-Crystal Phases of a Doped Mott Insulator, Nature (London) 393, 550 (1998).

[7] E. Fradkin and S. A. Kivelson, Liquid-Crystal Phases of Quantum Hall Systems, Phys. Rev. B 59, 8065 (1999).

[8] M. M. Fogler, A. A. Koulakov, and B. Shklovskii, Ground State of a Two-Dimensional Electron Liquid in a Weak Magnetic Field, Phys. Rev. B 54, 1853 (1996).

[9] R. Moessner and J. T. Chalker, Exact Results for Interacting Electrons in High Landau Levels, Phys. Rev. B 54, 5006 (1996).

[10] A. A. Koulakov, M. M. Fogler, and B. I. Shklovskii, Charge Density Wave in Two-Dimensional Electron Liquid in Weak Magnetic Field, Phys. Rev. Lett. 76, 499 (1996).

[11] E. Fradkin, S. A. Kivelson, E. Manousakis, and K. Nho, Nematic Phase of the Two-Dimensional Electron Gas in a Magnetic Field, Phys. Rev. Lett. 84, 1982 (2000).

[12] E. Fradkin, S. A. Kivelson, M. J. Lawler, J. P. Eisenstein, and A. P. Mackenzie, Nematic Fermi Fluids in Condensed Matter Physics, Annu. Rev. Condens. Matter Phys. 1, 153 (2010).

[13] M. P. Lilly, K. B. Cooper, J. P. Eisenstein, L. N. Pfeiffer, and K. W. West, Evidence for an Anisotropic State of TwoDimensional Electrons in High Landau Levels, Phys. Rev. Lett. 82, 394 (1999).

[14] R. R. Du, D. C. Tsui, H. L. Störmer, L. N. Pfeiffer, K. W. Baldwin, and K. W. West, Strongly Anisotropic Transport in Higher Two-Dimensional Landau Levels, Solid State Commun. 109, 389 (1999).

[15] W. Pan, A. Serafin, J. S. Xia, L. Yin, N. S. Sullivan, K. W. Baldwin, K.W. West, L. N. Pfeiffer, and D. C. Tsui, Competing Quantum Hall Phases in the Second Landau Level in the Low-Density Limit, Phys. Rev. B 89, 241302(R) (2014).

[16] Q. Qian, J. Nakamura, S. Fallahi, G. C. Gardner, and M. J. Manfra, Possible Nematic to Smectic Phase Transition in a Two-Dimensional Electron Gas at Half-Filling, Nat. Commun. 8, 1536 (2017).

[17] M. Levin, B. I. Halperin, and B. Rosenow, Particle-Hole Symmetry and the Pfaffian State, Phys. Rev. Lett. 99, 236806 (2007).

[18] S.-S. Lee, S. Ryu, C. Nayak, and M. P. A. Fisher, ParticleHole Symmetry and the $\nu=\frac{5}{2}$ Quantum Hall State, Phys. Rev. Lett. 99, 236807 (2007).
[19] C. Wang, A. Vishwanath, and B. I. Halperin, Topological Order from Disorder and the Quantized Hall Thermal Metal: Possible Applications to the $\nu=5 / 2$ State, Phys. Rev. B 98, 045112 (2018).

[20] D. F. Mross, Y. Oreg, A. Stern, G. Margalit, and M. Heiblum, Theory of Disorder-Induced Half-Integer Thermal Hall Conductance, Phys. Rev. Lett. 121, 026801 (2018).

[21] B. Lian and J. Wang, Theory of the Disordered $\nu=\frac{5}{2}$ Quantum Thermal Hall State: Emergent Symmetry and Phase Diagram, Phys. Rev. B 97, 165124 (2018).

[22] W. Pan, R. R. Du, H. L. Störmer, D. C. Tsui, L. N. Pfeiffer, K. W. Baldwin, and K.W. West, Strongly Anisotropic Electronic Transport at Landau Level Filling Factor $\nu=$ $9 / 2$ and $\nu=5 / 2$ under Tilted Magnetic Field, Phys. Rev. Lett. 83, 820 (1999).

[23] M. P. Lilly, K. B. Cooper, J. P. Eisenstein, L. N. Pfeiffer, and K. W. West, Anisotropic States of Two-Dimensional Electron Systems in High Landau Levels: Effect of an In-Plane Magnetic Field, Phys. Rev. Lett. 83, 824 (1999).

[24] B. Friess, V. Umansky, L. Tiemann, K. von Klitzing, and J. H. Smet, Probing the Microscopic Structure of Stripe Phase at Filling Factor 5/2, Phys. Rev. Lett. 113, 076803 (2014).

[25] X. Shi, W. Pan, K. W. Baldwin, K. W. West, L. N. Pfeiffer, and D. C. Tsui, Impact of the Modulation Doping Layer on the $\nu=5 / 2$ Anisotropy, Phys. Rev. B 91, 125308 (2015).

[26] J. Xia, V. Cvicek, J. P. Eisenstein, L. N. Pfeiffer, and K. W. West, Tilt-Induced Anisotropic to Isotropic Phase Transition at $\nu=$ 5/2, Phys. Rev. Lett. 105, 176807 (2010).

[27] Y. Liu, S. Hasdemir, M. Shayegan, L. N. Pfeiffer, K. W. West, and K. W. Baldwin, Evidence for a $\nu=5 / 2$ Fractional Quantum Hall Nematic State in Parallel Magnetic Fields, Phys. Rev. B 88, 035307 (2013).

[28] N. Samkharadze, K. A. Schreiber, G. C. Gardner, M. J. Manfra, E. Fradkin, and G. A. Csáthy, Observation of a Transition from a Topologically Ordered to a Spontaneously Broken Symmetry Phase, Nat. Phys. 12, 191 (2016).

[29] K. A. Schreiber, N. Samkharadze, G. C. Gardner, R. R. Biswas, M. J. Manfra, and G. A. Csáthy, Onset of Quantum Criticality in the Topological-to-Nematic Transition in a Two-Dimensional Electron Gas at Filling Factor $\nu=5 / 2$, Phys. Rev. B 96, 041107(R) (2017).

[30] K. A. Schreiber, N. Samkharadze, G. C. Gardner, Y. Lyanda-Geller, M. J. Manfra, L. N. Pfeiffer, K. W. West, and G. A. Csáthy, Electron-Electron Interactions and the Paired-to-Nematic Quantum Phase Transition in the Second Landau Level, Nat. Commun. 9, 2400 (2018).

[31] K. Lee, J. Shao, E.-A. Kim, F. D. M. Haldane, and E. H. Rezayi, Pomeranchuk Instability of Composite Fermi Liquids, Phys. Rev. Lett. 121, 147601 (2018).

[32] J. Xia, J. P. Eisenstein, L. N. Pfeiffer, and K. W. West, Evidence for a Fractionally Quantized Hall State with Anisotropic Longitudinal Transport, Nat. Phys. 7, 845 (2011).

[33] E. Berg, E. Fradkin, S. A. Kivelson, and J. M. Tranquada, Striped Superconductors: How Spin, Charge and Superconducting Orders Intertwine in the Cuprates, New J. Phys. 11, 115004 (2009).

[34] E. Fradkin, S. A. Kivelson, and J. M. Tranquada, Colloquium: Theory of Intertwined Orders in High Temperature Superconductors, Rev. Mod. Phys. 87, 457 (2015). 
[35] E. Berg, E. Fradkin, E.-A. Kim, S. A. Kivelson, V. Oganesyan, J. M. Tranquada, and S. C. Zhang, Dynamical Layer Decoupling in a Stripe-Ordered High- $T_{c}$ Superconductor, Phys. Rev. Lett. 99, 127003 (2007).

[36] F. Wilczek, Magnetic Flux, Angular Momentum, and Statistics, Phys. Rev. Lett. 48, 1144 (1982).

[37] J. K. Jain, Composite-Fermion Approach for the Fractional Quantum Hall Effect, Phys. Rev. Lett. 63, 199 (1989).

[38] A. López and E. Fradkin, Fractional Quantum Hall Effect and Chern-Simons Gauge Theories, Phys. Rev. B 44, 5246 (1991).

[39] B. I. Halperin, P. A. Lee, and N. Read, Theory of the HalfFilled Landau Level, Phys. Rev. B 47, 7312 (1993).

[40] X. Wan and K. Yang, Striped Quantum Hall State in a HalfFilled Landau Level, Phys. Rev. B 93, 201303(R) (2016).

[41] Md. Shafayat Hossain, M. K. Ma, Y. J. Chung, L. N. Pfeiffer, K. W. West, K. W. Baldwin, and M. Shayegan, Unconventional Anisotropic Even-Denominator Fractional Quantum Hall State in a System with Mass Anisotropy, Phys. Rev. Lett. 121, 256601 (2018).

[42] E. Berg, E. Fradkin, and S. A. Kivelson, Charge 4e Superconductivity from Pair Density Wave Order in Certain High Temperature Superconductors, Nat. Phys. 5, 830 (2009).

[43] K. Musaelian and R. Joynt, Broken Rotation Symmetry in the Fractional Quantum Hall System, J. Phys. Condens. Matter 8, L105 (1996).

[44] L. Balents, Spatially Ordered Fractional Quantum Hall States, Europhys. Lett. 33, 291 (1996).

[45] M. Mulligan, C. Nayak, and S. Kachru, Isotropic to Anisotropic Transition in a Fractional Quantum Hall State, Phys. Rev. B 82, 085102 (2010).

[46] M. Mulligan, C. Nayak, and S. Kachru, Effective Field Theory of Fractional Quantized Hall Nematics, Phys. Rev. B 84, 195124 (2011).

[47] J. Maciejko, B. Hsu, S. A. Kivelson, Y. J. Park, and S. L. Sondhi, Field Theory of the Quantum Hall Nematic Transition, Phys. Rev. B 88, 125137 (2013).

[48] Y. You, G. Y. Cho, and E. Fradkin, Theory of Nematic Fractional Quantum Hall States, Phys. Rev. X 4, 041050 (2014).

[49] D. X. Nguyen, A. Gromov, and D. T. Son, Fractional Quantum Hall Systems Near Nematicity: Bimetric Theory, Composite Fermions, and Dirac Brackets, Phys. Rev. B 97, 195103 (2018).

[50] D. T. Son, Is the Composite Fermion a Dirac Particle?, Phys. Rev. X 5, 031027 (2015).

[51] M. Sitte, A. Rosch, J. S. Meyer, K. A. Matveev, and M. Garst, Emergent Lorentz. Symmetry with Vanishing Velocity in a Critical Two-Subband Quantum Wire, Phys. Rev. Lett. 102, 176404 (2009).

[52] J. Toner and D. R. Nelson, Smectic, Cholesteric, and Rayleigh-Benard Order in Two Dimensions, Phys. Rev. B 23, 316 (1981).

[53] D. G. Barci and E. Fradkin, Role of Nematic Fluctuations in the Thermal Melting of Pair-Density-Wave Phases in TwoDimensional Superconductors, Phys. Rev. B 83, 100509(R) (2011).

[54] Y. Imry and S. K. Ma, Random-Field Instability of the Ordered State of Continuous Symmetry, Phys. Rev. Lett. 35, 1399 (1975).
[55] B. M. McCoy and T. T. Wu, Theory of a Two-Dimensional Ising Model with Random Impurities. I. Thermodynamics, Phys. Rev. 176, 631 (1968).

[56] R. Shankar and G. Murthy, Nearest-Neighbor Frustrated Random-Bond Model in $d=2$ : Some Exact Results, Phys. Rev. B 36, 536 (1987).

[57] D. S. Fisher, Random Transverse Field Ising Spin Chains, Phys. Rev. Lett. 69, 534 (1992).

[58] D. S. Fisher, Critical Behavior of Random Transverse-Field Ising Spin Chains, Phys. Rev. B 51, 6411 (1995).

[59] N. Read and D. Green, Paired States of Fermions in Two Dimensions with Breaking of Parity and Time-Reversal Symmetries and the Fractional Quantum Hall Effect, Phys. Rev. B 61, 10267 (2000).

[60] D. F. Agterberg and H. Tsunetsugu, Dislocations and Vortices in Pair-Density-Wave Superconductors, Nat. Phys. 4, 639 (2008).

[61] A. Himeda, T. Kato, and M. Ogata, Stripe States with Spatially Oscillating $d$-Wave Superconductivity in the TwoDimensional $t-t^{\prime}-J$ Model, Phys. Rev. Lett. 88, 117001 (2002).

[62] M. Raczkowski, M. Capello, D. Poilblanc, R. Frésard, and A. M. Oleś, Unidirectional $d$-Wave Superconducting Domains in the Two-Dimensional $t-J$ Model, Phys. Rev. B 76, 140505(R) (2007).

[63] Y. Wang, D. F. Agterberg, and A. Chubukov, Coexistence of Charge-Density-Wave and Pair-Density-Wave Orders in Underdoped Cuprates, Phys. Rev. Lett. 114, 197001 (2015).

[64] P. A. Lee, Amperean Pairing and the Pseudogap Phase of Cuprate Superconductors, Phys. Rev. X 4, 031017 (2014).

[65] L. Radzihovsky, Fluctuations and Phase Transitions in Larkin-Ovchinnikov Liquid-Crystal States of a PopulationImbalanced Resonant Fermi Gas, Phys. Rev. A 84, 023611 (2011).

[66] D. F. Agterberg, P. M. R. Brydon, and C. Timm, Bogoliubov Fermi Surfaces in Superconductors with Broken TimeReversal Symmetry, Phys. Rev. Lett. 118, 127001 (2017).

[67] E. Hassinger, P. Bourgeois-Hope, H. Taniguchi, S. R. de Cotret, G. Grissonnanche, M.S. Anwar, Y. Maeno, N. Doiron-Leyraud, and L. Taillefer, Vertical Line Nodes in the Superconducting Gap Structure of $\mathrm{Sr}_{2} \mathrm{RuO}_{4}$, Phys. Rev. X 7, 011032 (2017).

[68] A. Marchenkov, R. W. Simmonds, S. Backhaus, A. Loshak, J. C. Davis, and R. E. Packard, Bi-state Superfluid ${ }^{3}$ he Weak Links and the Stability of Josephson $\pi$ States, Phys. Rev. Lett. 83, 3860 (1999).

[69] A. Yu. Kitaev, Unpaired Majorana Fermions in Quantum Wires, Phys. Usp. 44, 131 (2001).

[70] D. A. Ivanov, Non-Abelian Statistics of Half-Quantum Vortices in $p$-Wave Superconductors, Phys. Rev. Lett. 86, 268 (2001).

[71] E. Berg, E. Fradkin, and S. A. Kivelson, Theory of the Striped Superconductor, Phys. Rev. B 79, 064515 (2009).

[72] S. Ryu, A. P. Schnyder, A. Furusaki, and A. W. W. Ludwig, Topological Insulators and Superconductors: Tenfold Way and Dimensional Hierarchy, New J. Phys. 12, 065010 (2010).

[73] J. M. Ziman, Principles of the Theory of Solids, 2nd ed. (Cambridge University Press, Cambridge, England, 1979). 
[74] For the rest of the work, we use "band" and "band topology" to refer to those for the BdG Hamiltonian.

[75] S. Kobayashi, K. Shiozaki, Y. Tanaka, and M. Sato, Topological Blount's Theorem of Odd-Parity Superconductors, Phys. Rev. B 90, 024516 (2014).

[76] Y. X. Zhao, A. P. Schnyder, and Z. D. Wang, Unified Theory of PT and CP Invariant Topological Metals and Nodal Superconductors, Phys. Rev. Lett. 116, 156402 (2016).

[77] T. Bzdušek and M. Sigrist, Robust Doubly Charged Nodal Lines and Nodal Surfaces in Centrosymmetric Systems, Phys. Rev. B 96, 155105 (2017).

[78] O. Türker and S. Moroz, Weyl Nodal Surfaces, Phys. Rev. B 97, 075120 (2018).

[79] P. M. R. Brydon, D. F. Agterberg, H. Menke, and C. Timm, Bogoliubov Fermi Surfaces: General Theory, Magnetic Order, and Topology, Phys. Rev. B 98, 224509 (2018).

[80] D. F. Mross, J. Alicea, and O. I. Motrunich, Explicit Derivation of Duality between a Free Dirac Cone and Quantum Electrodynamics in $(2+1)$ Dimensions, Phys. Rev. Lett. 117, 016802 (2016).

[81] W. P. Su, J. R. Schrieffer, and A. J. Heeger, Solitons in Polyacetylene, Phys. Rev. Lett. 42, 1698 (1979).

[82] J. M. Murray and O. Vafek, Majorana Bands, Berry Curvature, and Thermal Hall Conductivity in the Vortex State of a Chiral p-Wave Superconductor, Phys. Rev. B 92, 134520 (2015).

[83] M. Tinkham, Introduction to Superconductivity (McGrawHill, New York, 1996).

[84] C. L. Kane, A. Stern, and B. I. Halperin, Pairing in Luttinger Liquids and Quantum Hall States, Phys. Rev. X 7, 031009 (2017).

[85] B. I. Halperin, Theory of the Quantized Hall Conductance, Helv. Phys. Acta 56, 75 (1983).
[86] J. M. Kosterlitz and D. J. Thouless, Order, Metastability and Phase Transitions in Two-Dimensional Systems, J. Phys. C 6, 1181 (1973).

[87] D. R. Nelson and B. I. Halperin, Dislocation-Mediated Melting in Two Dimensions, Phys. Rev. B 19, 2457 (1979).

[88] A. P. Young, Melting and the Vector Coulomb Gas in Two Dimensions, Phys. Rev. B 19, 1855 (1979).

[89] C. Wang, Braiding Statistics and Classification of TwoDimensional Charge-2m Superconductors, Phys. Rev. B 94, 085130 (2016).

[90] S. A. Parameswaran, S. A. Kivelson, S. L. Sondhi, and B. Z. Spivak, Weakly Coupled Pfaffian as a Type I Quantum Hall Liquid, Phys. Rev. Lett. 106, 236801 (2011).

[91] S. A. Parameswaran, S. A. Kivelson, E. H. Rezayi, S. H. Simon, S. L. Sondhi, and B.Z. Spivak, Typology for Quantum Hall Liquids, Phys. Rev. B 85, 241307(R) (2012).

[92] Z. Zhu, I. Sodemann, D. N. Sheng, and L. Fu, AnisotropyDriven Transition from the Moore-Read State to Quantum Hall Stripes, Phys. Rev. B 95, 201116(R) (2017).

[93] S. K. Paul and A. Khare, Charged Vortices in an Abelian Higgs Model with Chern-Simons Term, Phys. Lett. B 174, 420 (1986).

[94] S. D. Edkins, A. Kostin, K. Fujita, A. P. Mackenzie, H. Eisaki, S.-I. Uchida, S. Sachdev, M. J. Lawler, E.-A. Kim, J. C. S. Davis, and M. H. Hamidian, Magnetic-Field Induced Pair Density Wave State in the Cuprate Vortex Halo, arXiv:1802.04673.

[95] Y. Wang, S. D. Edkins, M. H. Hamidian, J. C. S. Davis, E. Fradkin, and S. A. Kivelson, Pair Density Waves in Superconducting Vortex Halos, Phys. Rev. B 97, 174510 (2018).

[96] M. Barkman, A. A. Zyuzin, and E. Babaev, Anti-chiral and Nematicity-Wave Superconductivity, arXiv:1811.10594. 